\title{
Advanced liposome-loaded scaffolds for therapeutic and tissue engineering applications
}

\section{Cheng, Ruoyu}

2020-02

Cheng , R , Liu , L , Xiang , Y , Lu , Y , Deng , L , Zhang , H , Santos , H A \& Cui , W 2020 , ' Advanced liposome-loaded scaffolds for therapeutic and tissue engineering applications ' , Biomaterials , vol. 232 , 119706 . https://doi.org/10.1016/j.biomaterials.2019.119706

http://hdl.handle.net/10138/313174

https://doi.org/10.1016/j.biomaterials.2019.119706

draft

Downloaded from Helda, University of Helsinki institutional repository.

This is an electronic reprint of the original article.

This reprint may differ from the original in pagination and typographic detail.

Please cite the original version. 


\section{Journal Pre-proof}

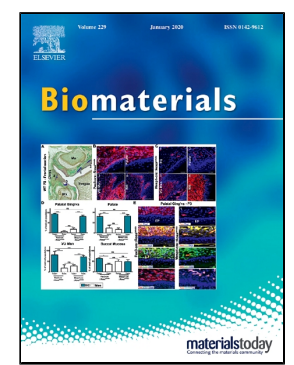

Ruoyu Cheng, Lili Liu, Yi Xiang, Yong Lu, Lianfu Deng, Hongbo Zhang, Hélder A.

Santos, Wenguo Cui

PII:

S0142-9612(19)30824-5

DOI: https://doi.org/10.1016/j.biomaterials.2019.119706

Reference:

JBMT 119706

To appear in:

Biomaterials

Received Date:

09 May 2019

Accepted Date:

18 December 2019

Please cite this article as: Ruoyu Cheng, Lili Liu, Yi Xiang, Yong Lu, Lianfu Deng, Hongbo Zhang, Hélder A. Santos, Wenguo Cui, Advanced liposome-loaded scaffolds for therapeutic and tissue engineering applications, Biomaterials (2019), https://doi.org/10.1016/j.biomaterials.2019.119706

This is a PDF file of an article that has undergone enhancements after acceptance, such as the addition of a cover page and metadata, and formatting for readability, but it is not yet the definitive version of record. This version will undergo additional copyediting, typesetting and review before it is published in its final form, but we are providing this version to give early visibility of the article. Please note that, during the production process, errors may be discovered which could affect the content, and all legal disclaimers that apply to the journal pertain.

(C) 2019 Published by Elsevier. 


\section{Advanced liposome-loaded scaffolds for therapeutic and tissue engineering applications}

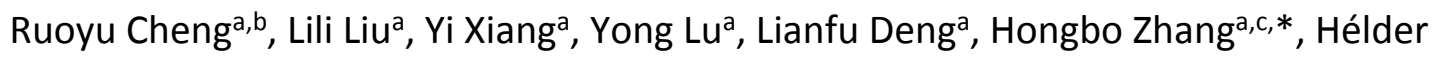

$$
\text { A. Santos }{ }^{b, d}, * \text {, Wenguo Cuia,* }
$$

a Shanghai Key Laboratory for Prevention and Treatment of Bone and Joint Diseases, Shanghai Institute of Traumatology and Orthopaedics, Ruijin Hospital, Shanghai Jiao Tong University School of Medicine, 197 Ruijin second Road, Shanghai 200025, P. R. China

${ }^{b}$ Drug Research Program, Division of Pharmaceutical Chemistry and Technology, Faculty of Pharmacy, University of Helsinki, Fl-00014 Helsinki, Finland

c Department of Pharmaceutical Sciences Laboratory and Turku Bioscience Center, Åbo Akademi University, Turku, 20520, Finland

d Helsinki Institute of Life Science (HiLIFE), University of Helsinki, Fl-00014 Helsinki, Finland

*Corresponding authors: hongbo.zhang@abo.fi (Hongbo Zhang);

helder.santos@helsinki.fi (Hélder A.Santos); wgcui80@hotmail.com (Wenguo Cui); 


\begin{abstract}
Liposome is one of the most commonly used drug delivery systems in the world, due to its excellent biocompatibility, satisfactory ability in controlling drug release, and passive targeting capability. However, some drawbacks limit the application of liposomes in clinical, such as problems in transporting, storing, and difficulties in maintaining the drug concentration in the local area. Scaffolds usually are used as implants to supply certain mechanical supporting to the defective area or utilized as diagnosis and imaging methods. But, in general, unmodified scaffolds show limited abilities in promoting tissue regeneration and treating diseases. Therefore, liposome-scaffold composite systems are designed to take advantages of both liposomes' biocompatibility and scaffolds' strength to provide a novel system that is more suitable for clinical applications. This review introduces and discusses different types of liposomes and scaffolds, and also the application of liposome-scaffold composite systems in different diseases, such as cancer, diabetes, skin-related diseases, infection and human immunodeficiency virus, and in tissue regeneration like bone, teeth, spinal cord and wound healing.
\end{abstract}

KEYWORDS: Liposomes; scaffolds; tissue regeneration; diseases treatment 


\section{Introduction}

Liposomes are closed vesicles with bilayer structure that is formed by dispersing amphipathic molecule, such as phospholipid, into water. Due to the hydrophobic tail and the hydrophilic head section, these tails tend to aggregate to avoid contacting with water and these heads are likely to expose to water[1]. The special bilayer structure makes these carriers can load hydrophilic drug in the internal water phase and the hydrophobic drug in the bilayer.

In the early stage (1960), liposomes were regarded as a research model of cell membrane, while nowadays they are mainly used as drug carriers to deliver proteins, growth factors and other chemical drugs. As drug delivery carriers, liposomes have various advantages, such as (1) passive targeting via enhanced permeability and retention effect in tumor area, (2) excellent ability in controlling drug release, (3) decreased drug side effects, and (4) improved drug stability, which make them effective in delivering drugs, vaccines, imaging, cosmetics, etc.[2]. However, liposomes have also drawbacks that limit their clinical applications, such as (1) challenges in transporting liquid formulation, (2) insufficient mechanical properties, and (3) inability to maintain certain drug concentration in the local area. Therefore, certain improvements are needed to offer more suitable liposomes for clinical applications.

Tissue engineering, also known as regenerative medicine, aims to develop bioactive implants that can repair or ameliorate the structure and functionality of tissue defects. In tissue engineering, there are three critical elements, cells, scaffolds and bioactive factors[3]. Among them, scaffolds, which can diagnose, repair or improve the functionality in tissue and organ, are regarded as core materials and have attracted a considerable attention in research. Moreover, scaffolds can provide unique features that cannot be achieved by drugs [4], such as (1) provide certain mechanical supporting in defect areas, (2) stimulate cells' differentiation by regulating the Youngs' modulus, and (3) execute, raise or replace some lost functions caused by diseases or impairments. Scaffolds can provide mechanical support and 
appropriate environment for cell proliferation, and control the flow of blood or other body fluid, which functions as fillers. It is difficult to achieve the repairing goals in serious injuries portion or other clinical requirements when scaffolds are utilized without any drugs or cells.

Recently, drug-loaded scaffolds were proved to have both therapeutic effects brought by drugs and mechanical support supplied by materials. However, these drug-loaded scaffolds still have some problems, e.g., (1) limited choices at drug types, (2) burst drug release, and (3) difficulty in maintaining certain drug concentration in local areas. Therefore, based on the advantages of liposomes (drug carriers) and the benefits of scaffolds (mechanical supporting), it is reasonable to believe that combining liposomes with scaffolds can help drugs maintain certain concentration in situ for a long period, and the composite systems can provide more functionalities. For example, liposomes-modified scaffolds have more choices at loaded drug types, and in addition, drugs carried in liposomes can provide the composite scaffold capabilities in promoting regeneration in defect areas. Moreover, controlled and responsive drug release in scaffolds can be achieved by using functional liposomes.

Herein, this review introduces and discusses different types of liposomes and scaffolds, and also the application of liposome-scaffold composite systems in different diseases, such as cancer, diabetes, skin-related diseases, infection and human immunodeficiency virus, and in tissue regeneration like bone, teeth, spinal cord and wound healing.

\section{Liposomes}

In 1960s, Bangham[5] observed the specific behaviors of lipids by transmission electron microscope (TEM). When he dispersed lipids into water, he found a kind of vesicle filled with water in the inner core that was similar to the structure of cells. According to the images collected from the mixture of lipids and water, he named this kind of material as liposomes. The self-assembling core-shell vesicle structure 
enables liposomes to carry both hydrophobic and hydrophilic drugs, which is regarded as the most ancient nano-drug delivery system. Since the first liposomes were fabricated, researchers have done a series of investigations about the biophysical and biochemical properties of liposomes. For example, liposomes were utilized as a model to explore cell membranes, these nanoparticles were also used in the areas of drug delivery[6], diagnostics[7], imaging[8], food and cosmetic industries [9, 10], molecular biology[11], biochemistry[12], and microfluidics[13].

In this review, we will mainly focus on the drug delivery capability of liposomes and discuss the special functionalities and limitations in application.

\subsection{Drug-loaded liposomes}

Liposomes have been widely investigated as drug carriers, from the approved amphotericin B liposome in 1990 to the successful Onivyde ${ }^{\top M}$ in 2015. As drug carriers, liposomes have several advantages, for instance, they can carry various types of drugs, specifically, the internal water core can load hydrophilic drugs and the bilayers can carry hydrophobic ones. Additionally, liposomes exhibit the ability in controlling the drug release and decreasing the side effect of drugs. Furthermore, liposomes also show advantages in low toxicity, non-immunogenic and biodegradability. The phospholipid bilayer can be regarded as satisfactory platforms that can be modified with diverse ligands to achieve the goal of targeting delivery. Furthermore, the stability and efficacy of biological products can also be improved by utilizing liposomes as vehicles.

\subsubsection{Delivering small molecule hydrophilic drugs}

Several methods, such as reverse evaporation, $\mathrm{pH}$ gradient, ammonium sulfate gradient and repeated freeze thawing, are usually applied in the process of preparing small hydrophilic drugs-loaded liposomes. For example, Takeuchi et al.[14] fabricated polyborane encapsulated liposomes though $\mathrm{pH}$ gradient or reverse-phase evaporation, then they found that for the encapsulation efficiency of the liposome 
prepared using the $\mathrm{pH}$ gradient was twice as high as that prepared, using reverse-phase evaporation. Additionally, they observed that boron concentration of the polyborane encapsulated liposomes prepared using the $\mathrm{pH}$ gradient achieved $110-150 \mu \mathrm{g} / \mathrm{g}$ of tumor tissue.

\subsubsection{Delivering small molecule hydrophobic drugs}

Usually the film dispersion method is one of the simplest ways to fabricate lipid drug-loaded liposomes. For example, $\mathrm{Fu}$ et al.[15] fabricated novel temperature-sensitive liposomes loading paclitaxel (PTX-TSL) by using a film dispersion method. The encapsulation efficiency of K237-PTX-TSL was 94.23 $( \pm 0.76) \%$. The particle diameter was $88.3 \pm 4.7 \mathrm{~nm}$. Liao et al.[16] prepared Iridium (III) complex-loaded liposomes by thin-film dispersion and ultrasonic method. The average particle size, polydispersity index, zeta-potential, encapsulation efficiency, and drug loading are $112.57 \pm 1.15 \mathrm{~nm}, 0.19 \pm 0.02,-10.66 \pm 0.61 \mathrm{mV}, 94.71( \pm 3.21) \%$, and $4.71( \pm 0.41) \%$, respectively.

\subsubsection{Delivering gene drugs}

As non-viral delivery system, one of the most popular functions of liposomes is transfection. By using tripeptide-based lipid (CDO) as a model lipid and sucrose esters (SEs) as helper lipids, a series of liposomes were prepared by Zhao et al.[17] The transfection efficiencies of the liposomes, containing SEs with hydrophiliclipophilic balance (HLB) value of 6 were superior to other liposomes in HeLa, MCF-7, $\mathrm{NCl}-\mathrm{H} 460$, and $\mathrm{A} 549$ tumor cells.

\subsubsection{Delivering growth factors}

Growth factors can regulate the cell behaviors. For example, keratinocyte growth factor (KGF) is effective to treat ulcerative colitis. But this growth factor fails to be stable and specific distribution toward inflamed bowel. Zhao et al.[18] fabricated KGF-loaded liposomes with a high encapsulation efficiency of $95.3( \pm 0.72) \%$, PDI of 0.18 , and zeta-potential of $-2.37 \pm 0.14 \mathrm{mV}$. 


\subsubsection{Delivering proteins}

Comparing to small molecule drugs, proteins have more specific functions, higher bioactivity and lower toxicity, which is more beneficial for clinical applications. Liposomes have also been used for protein delivery. For example, Dapergolas et al.[19] fabricated insulin-loaded liposomes to treat diabetes rats. Yamada et al.[20] utilized liposomes to deliver coenzyme Q10 to improve its cellular uptake, which provides a novel strategy in treating mitochondria-related diseases.

\subsection{Functionalized liposomes}

\subsubsection{Long circulating liposomes}

Surface modification of the scaffold with polyethylene glycol (PEG), phosphatidylinositol (PI) or ganglioside can bring dense conformational cloud to the liposome systems. This three-dimensional conformation can protect liposomes from opsonin recognition in blood and reduce rapid phagocytosis or uptake in reticuloendothelial system. Therefore, the clearing rate of liposomes can be decreased in vivo and the circulation time in blood can be extended, leading to the prolong drug circulation in blood.[21]

\subsubsection{Thermosensitive liposomes}

Typically, thermosensitive liposomes (TSL) consist of thermo-responsive materials, such as dipalmitoyl phosphatidylcholine (DPPC) and distearoyl phosphatidylcholine (DSPC), which can transform from a solid gel to a highly permeable liquid structure with increasing temperature. Usually, this kind of liposomes consist of special lipid materials with slightly higher phase transition temperature than the body temperature, and the drug release in this case is mediated by temperature[22, 23$]$.

\subsection{3 pH-Sensitive liposomes}

Some common $\mathrm{pH}$-sensitive materials, such as dioleoyl phosphatidylethanolamine 
(DOPEA)[24, 25], N-(4-carboxybenzyl)-N,N-dimethyl-2,3-bis(oleoyloxy)propan-1aminium (DOBAQ), are widely used in the fabrication of $\mathrm{pH}$-responsive liposomes. For liposomes prepared with these materials, when the local area exhibits slightly acid $\mathrm{pH}(\mathrm{pH}<6)$, the carboxyl in fatty acid is protonated, causing the formation of a hexagonal phase and the fusion between the cell membranes and liposomes, which benefits the intracellular drug delivery. The microenvironment in the tumor area is usually acid, additionally, acid pH-responsive liposomes have been widely investigated in targeting drug delivery for cancer therapy.

\subsubsection{Magnetic liposomes}

During the process of liposome preparation, magnetic materials can be added to endow the system with magnetic response [26]. When these intelligent carriers are injected into body, the movement of these liposomes can be controlled by applied an external magnetic field to guide these nanoparticles to the local area. These vehicles are proved to improve the specificity of the therapy and the accuracy of diagnosis (e.g., by magnetic resonance imaging)[27]. Moreover, liposomes can also contain multifunction, including imaging, diagnosis to achieve high efficiency with low adverse effect $[28,29]$.

\subsubsection{Active targeting liposomes}

Active targeting can be achieved by specific binding between receptors and ligands, ligands could be some common compounds, for example, antibodies, Fab fragments, DNA or RNA sequences, transferrin, and peptides. Generally, these ligands can be used to fabricate active targeting liposomes to get more efficient therapy. [30, 31].

\subsection{Summary}

As novel drug carriers, liposomes have achieved significant success in clinical applications, such as amphotericin B@liposome, doxorubicin@liposome, and daunorubicin@liposome. However, there are still some disadvantages in this drug 
delivery system, for example, the challenge in mass production, poor reproducibility, high production costs, the leakage of loading drugs, and the fusion among liposomes. These drawbacks largely limit the application of liposomes in clinic.

\section{Scaffolds for drug loading}

Currently, scaffolds are widely used for diagnosis, tissue regeneration and function restoration in some organs. Basically, there are mainly three types of scaffolds: metal scaffolds, such as alkali metal scaffolds and some alloy scaffolds; inorganic scaffolds, likes bioactive glass and hydroxyapatite; and organic scaffolds, e.g. polymeric based scaffolds. These scaffolds can supply necessary mechanical support to the local defect area and provide suitable microenvironment for cell proliferation, and these materials can also control the flow of blood and body fluid. However, scaffolds themselves have low therapeutic effect in local area, thus they cannot meet various demands in clinic. Therefore, fabricating drug-loaded scaffolds is necessary for expanding the scaffolds' clinical applications. In this section we introduce different types of scaffolds and the method of drug-loading.

\subsection{Metal scaffolds}

As a result, of the excellent mechanical strength, toughness and anti-fatigue performance, metal scaffolds have been widely used in tissue engineering. For example, these metal materials have be used to repair or replace the lesioned or partly worn teeth[32], bones[33], joints or other tissues. These materials can also be regarded as cardiovascular stents[34, 35], artificial heart valve[36], etc. Moreover, these metal scaffolds can be produced in different shapes, such as needle, nail, intramedullary needle, crown, triangular nail[37], which is suitable for diverse applications in tissue engineering.

The most common materials for making metal scaffolds include medical precious metals and their alloys, for example, gold, silver, platinum and their alloys, 
medical stainless-steel[38], medical cobalt-based alloy[39], medical titanium alloy and medical titanium nickel alloy[40]. Some of these metals can be further modified to make them more functional. For example, Pan et al. [41] modified the surface of titanium implants by two mussel-derived bioactive peptides to achieve the goal of specific cell adhesion and osteogenicity. For these metal materials, the advantages to apply them in tissue regeneration are the efficiently mechanical strength supported by them, and the stable physicochemical properties. However, they also meet some challenges, for example, usually it is difficult to produce drug-loaded metal scaffolds.

\subsection{Inorganic scaffolds for drug loading}

Inorganic scaffolds own favorable biocompatibility, mechanical strength and can release some bioactive elements that are necessary in the process of regeneration, thus these scaffolds have been widely investigated. For example, bioactive glass is one type of inorganic scaffold that have been extensively utilized in promoting bone regeneration, because of their excellent biocompatibility, and potential ability in accelerating bone repairing[42], as shown in Figure 1. Hydroxyapatite is one of the most important complements in human bones. The hydroxyapatite based scaffold can not only supply certain mechanical support in the local area, but also constantly release some bioactive elements to the external environment, such as calcium, phosphorus, which are beneficial for tissue repair[43]. Bioinert ceramics like alumina ceramics, glass ceramics, single crystal ceramics, and zirconia ceramics have stable chemical properties[44]. Additionally, these inorganic implants can be further modified by some drugs to make them multifunctional. For example, Yao et al.[45] fabricated dexamethasone-loaded carbon nanotube (CNT), then they combined these nanoparticles with nano-hydroxyapatite/polyamide 66 porous scaffold to promote the differentiation of BMSCs into osteoblast.

\subsection{Organic scaffolds for drug-loading}

Most organic scaffolds are polymer scaffolds, according to the sources, these organic 
scaffolds can be further divided into synthetic polymeric scaffolds, such as polylactic acid (PLA), polycaprolactone (PCL), and poly-L-lactide lactone, and natural polymeric scaffolds, like gelatin, HA, chitosan, and cellulose.

\subsubsection{Synthetic polymeric scaffolds for drug loading}

Polyglycolic acid (PGA) is a biodegradable, thermoplastic polymer and the simplest linear, aliphatic polyester with a glass transition temperature between 35 and $40{ }^{\circ} \mathrm{C}$. The final degradation products of PGA are carbon dioxide and water, which can be eliminated by body metabolism. Usually, PGA fibers are particularly stiff with high strength and modulus ( $7 \mathrm{GPa})$. This synthetic polymer has been widely used in fabricating internal fixation of fracture, scaffolds for bone regeneration and tendon repair, suture material, etc. Dehnavi et al. [46]prepared a novel conduit based on polymer blend nanocomposites of PGA, collagen, and nanobioglass were prepared by electrospinning technique, which can have potential for nerve regeneration. To treat intervertebral disc degenerative disease, Abbushi et al.[47] filled the defected intervertebral disc area with hybrid scaffold containing PGA and HA. This scaffold was proved can not only supply efficient mechanical strength to the local area, but also to accelerate tissue regeneration.

Different from PGA's poor degradability, PLA can be gradually degraded to lactic acid, which will be further adsorbed by the body. Generally, the degradation half-life of PLA is around half to two years. Additionally, due to the low glass transition temperature (around $65^{\circ} \mathrm{C}$ ), PLA is also regarded as a candidate in researching shape memory polymer and 3D-printing technology. Due to these benefits, the applications of PLA in tissue engineering are explored by researchers. For example, Charlesharris et al.[48] investigated the capability of 3D porous scaffolds, containing PLA and calcium phosphate nanoparticles in mediating cell behaviors, during the process of bone regeneration. Chatzikyriakou et al.[49] fabricated composite materials by mixing PLA with $\beta$-tricalcium phosphate to promote skull repair. Thakur et al.[50] also dissolved lidocaine and mupirocin in the PLA solution, respectively. Then, they used double nozzle electrospinning technology to construct a complex fiber system, 
in this system, every single fiber contained these two different drugs, respectively. In the drug release experiments in vitro, because of the different release characteristics between the two drugs, staged drug release was observed in this system. PLA is also easy to be modified with other chemical compounds, which makes it become a novel polymer material for scaffold fabrication. For example, Murakami et al.[51] modified the end of PEG-PLA block compound with aldehyde, using a Schiff base reaction with amino compound to form a hydrogel in 2 seconds, which leads to generate a hydrogel with tissue adhesion ability and provide a novel application strategy for tissue engineering.

$\mathrm{PCL}$ is famous for soft texture, excellent extensibility and low temperature molding. This material has been regarded as carriers for controlling drug release, as scaffolds for culturing cells and tissues, and also surgical suture. For example, Louvrier et al. [52] evaluated the ability of DPSCs to colonize, proliferate and differentiate into functional odontoblast-like cells when cultured onto a polycaprolactone cone fabricated by jet-spraying and prototyped into a design similar to a gutta-percha cone. Alireza et al. [53]prepared a PCL/bioactive glass bone scaffold, they found that scaffolds sintered at $64.5^{\circ} \mathrm{C}$ for $100 \mathrm{~min}$ had optimal mechanical properties and 5 wt\% scaffolds behaved closer to bone.

PLGA is an FDA approved medical polymer that can be applied as anti-adhesion film and excipient in controlling drug release. Liu et al. [54] prepared a highly porous PLGA scaffolds with interconnected pore structures and well orientated microtubules, then the surface of scaffold was modified with air plasma for simultaneously tackling the dimensional shrinkage of PLGA scaffolds and improving scaffold-tissue integration. To reduce acidification by degradation of PLGA, Park et al. [55]incorporated magnesium hydroxide nanoparticles into porous polymer scaffold, this implant was proved can not only to effectively neutralize the acidic hydrolysate but also to minimize the structural disturbance of scaffolds.

\subsubsection{Natural polymer scaffolds for drug delivery}

Natural polymer scaffolds have been broadly applied in clinical applications, because 
of their abundant resources, excellent biocompatibility, biodegradability, low immunogenicity, and environmentally friendly.

Collagen is one of the most important components in the extracellular matrix of cartilage tissues. Recently, collagen-alginate was used as bioink for three dimensional cell printing based cartilage tissue engineering[56]. Achilli et al.[57] investigated the influence of $\mathrm{pH}$, ionic strength and temperature on the mechanical properties of collagen, further they designed a mechanical-controllable collagen hydrogel for vascular tissue engineering.

$\mathrm{HA}$ is a polymer polysaccharide consisting of glucuronic acid-N-acetylglucosamine disaccharide unit. HA widely distributes in various cell matrix and tissues. HA can be metabolized into glucosamine then fully absorbed by body. Taking advantage of this, Ehsan et al. [58] introduced an elastic, antimicrobial, and adhesive hydrogel comprised of methacrylated hyaluronic acid (MeHA) and an elastin-like polypeptide (ELP), which can be rapidly photo-cross-linked in situ for the regeneration and repair of different tissue. In addition, Kim et al.[59] fabricated a matrix metalloproteinase sensitive composite HA hydrogel by grafting two different peptides on $\mathrm{HA}$, and then they cultured human mesenchymal stem cells on the surface of this hydrogel. As a result, these cells showed relatively high level in the expression of osteogenic related genes.

Although, chitosan (CS) does not belong to the composition of human extracellular matrix, the structure and properties of CS are quite similar with amino dextran that is the main ingredient of extracellular matrix. In the area of tissue engineering, CS shows many advantages, such as outstanding biocompatibility, degradability, non-immunogenic, pyrogen-free reaction and ability in promoting wound healing. Based on abovementioned benefits, Frohbergh et al.[60] used electrospinning technology to fabricate an artificial periosteum that consisted of CS, hydroxyapatite and a natural bio-crosslinker Genipin. Co-culture this system with osteoblast, promising results were observed in the proliferation, differentiation and maturation of osteoblast. Recently, a biocomposite scaffolds containing chitosan (CS), nano-hydroxyapatite (nHAp) and nano-zirconium dioxide $\left(\mathrm{nZrO}_{2}\right)$ along with 
microRNA (miRNA) for bone tissue regeneration applications was reported [61].

Gelatin derives from partly degraded collagen in the connective tissue including skin, bones, muscle membrane, etc. These materials have been widely utilized in clinical treatments, due to their excellent biocompatibility and wide variety of sources. For example, Levett et al.[62] fabricated multifunctional scaffolds containing photo-crosslinking gelatin, $\mathrm{HA}$ and chondroitin sulfate to mimic the extracellular matrix for cartilage tissue engineering. Cheng et al.[63] also fabricated hydrogen-enhanced composite gelatin hydrogel modified with liposomes to deliver different types of drugs and promote bone regeneration, as shown in Figure 2.

\subsubsection{Composite polymer scaffolds for drug delivery}

Although, synthetic polymer scaffolds exhibit outstanding chemical stability, degradability, favorable mechanical property, controlled porosity and degradation rate, most of these materials are poor hydrophilicity with limited cell adhesion[64]. Moreover, the degradation products are usually acidic which can cause sterile inflammatory. And it is difficult to fully avoid solvent residue in synthetic scaffold, which will lead to a series of side effects in cells and causing inflammation and fibrosis in surrounding tissues. In contrast, a natural polymer usually has satisfactory biocompatibility and cytocompatibility. Furthermore, because these materials mainly derive from nature, the structure of a natural polymer is much similar with the main composition of the human extracellular matrix and the degradation production can be absorbed by human body. However, the short degradation period and unsatisfactory mechanical property limit the clinical application of some natural materials. Therefore, combining two or more different types of polymers which have complementary properties in a certain proportion will provide promising strategies in designing more favorable biomaterial-based scaffolds for clinical therapy.

Many efforts have been done by researchers to achieve this goal, for example, Du et al. [65] fabricated a heparin sodium and VEGF co-loaded bionic 3D scaffolds with CS and PCL. Furthermore, they used this composite implant to prevent generating local thrombus around the vascular implant with small diameter and 
promote the regeneration of vascular endothelial tissue in the local area, as shown in Figure 3. Dai et al. [66] constructed complex 3D scaffolds with controlled thickness by blending natural type I collagen with PLGA and applied it for the joint and cartilage tissue engineering. Kim et al. [67] utilized electrospinning to prepare scaffolds consisting of polyvinyl alcohol (PVA) and HA. Then these scaffolds' cell adhesion abilities were investigated. Results indicated that this implant provide a suitable platform for cell culture and tissue engineering. Meng et al. [68] prepared PLGA/CS composite scaffolds with phenylbutyric acid by random and directed electrospinning, respectively. Then the effects of CS proportion and the influence of electrospinning methods on the properties of drug release in vitro were explored in these fibers. Results revealed that the drug release rate increased with the raising chitosan content, because the addition of chitosan enhanced the hydrophilicity of the PLGA/chitosan composite scaffold. Additionally, aligned fibers showed lower drug release rate than that of randomly oriented scaffolds. Zhu et al. [69] utilized PEG-b-PCL amphiphilic block copolymer micelles to carry adriamycin through hydrophobic interaction. Then these micelles were combined with cyclodextrin solution to prepare supramolecular composite hydrogel which showed potent inhibition ability to bladder cancer in vitro.

\subsection{Summary}

Various types of biomaterial scaffolds provide the foundation for carrying different drugs or combining with several drug carriers. For example, most of hydrophilic drugs can be easily combined with many natural polymer scaffolds, and in contrast, hydrophobic drugs tend to combine with synthetic materials, such as PLA, PCL, PLGA, etc. Moreover, drugs-loaded carriers can combine with many types of biomaterials scaffolds, which means that most of these drug carriers can be loaded into various scaffolds, making the necessary foundation for further clinical applications. 


\section{The application of scaffolds combined with liposomes in tissue engineering}

As one of the FDA approved nanocarrier, during the last 30 years, liposomes have been investigated in diverse applications. Several properties are considered as main advantages to employ liposomes as drug carriers, such as satisfied biocompatibility, passive targeting ability, easily modified surface, etc. Due to these benefits, liposomes are combined with scaffold to make scaffold more functional, and at the same time, to take advantage from both sides and avoid drawbacks. For example, liposomes enable scaffold to have more choices in carrying drugs, regardless of the materials properties of the scaffolds. In addition, when liposomes are modified on the surface of implants or mixed into the matrix of scaffold, liposomes can be concentrated on the local area, which is difficult to achieve by intravenous injection.

Scaffold is defined as a biomaterial structure that serves as a substrate and guide for tissue repair and regeneration, which means that not only bulk materials, like bare-metal stent or titanium stick, but also nanomaterials, such as nanoparticles, carbon nanotubes, can be regarded as a part in scaffolds.

In this section, several types of scaffolds modified with liposomes are introduced to give an overview about how these composite systems function in treating diseases and their applications in tissue regeneration, are shown in Table 1.

\subsection{Metal scaffold combined with liposomes}

Metal scaffolds are mainly made of pure metal and alloy, which have excellent mechanical strength, toughness, anti-fatigue performance and outstanding conductivity in heat and electricity[70, 71]. However, these metal scaffolds have many limitations in meeting clinical demands, due to the simple functions. Therefore, recently, liposomes have been used to modify these metal scaffolds to meet the clinical requirements. For example, Antimisiaris et al.[72] modified nitinol 
stents with dexamethasone-loaded liposomes, this complex system exhibited 50.84 $( \pm 5.48) \%$ dexamethasone release in simulated urine after $48 \mathrm{~h}$ co-incubation. Additionally, no drug release can be observed in dry environment, which provides a novel strategy in controlling the drug release under the ureter environment. Different metal nanoparticles exhibit diverse characteristics that can be utilized in distinct applications. For example, $\mathrm{TiO}_{2}$ nanoparticles have photocatalytic performance, antibacterial ability and antitumor capability; $\mathrm{Fe}_{3} \mathrm{O}_{4}$ nanoparticles are usually used in performing magnetic resonance imaging, photothermal therapy, immunoassay and cell separation; and $\mathrm{ZnO}$ nanoparticles are frequently utilized in preparing biological sensors. Wang et al. [73] (Figure 4) modified liposomes with these three different metal nanoparticles to prepare a heterogeneous system that could be controlled by light, therefore this system can simultaneously deliver drug into the cell. Specifically, $\mathrm{Rh}-\mathrm{DOPC} / \mathrm{TiO}_{2}$ were internalized by endocytosis, $\mathrm{Rh}-\mathrm{DOPC} / \mathrm{SiO}_{2}$ and $\mathrm{Rh}-\mathrm{DOPC} / \mathrm{Fe}_{3} \mathrm{O}_{4}$ can also be internalized by HeLa cells. Some interesting phenomenon were also observed in this research, the phosphate in the lipid head group directly bonded with the metal oxide surface.

\subsection{Inorganic scaffold combined with liposomes}

Inorganic scaffolds usually consist of silicate, phosphate, borate, aluminate or sulfide, silicide, boride, phosphide, etc. Generally, most of the inorganic scaffolds, such as bioactive glass, hydroxyapatite, bio-inert ceramic, show favorable biocompatibility and acceptable mechanical strength. Moreover, these materials can also release some necessary elements to the surrounding environment in the process of tissue repair.

Wang et al.[74] (Figure 5) investigated different interaction between inverse phosphocholine lipids (CP) with $\mathrm{TiO}_{2}$ and $\mathrm{SiO}_{2}$, they found that $\mathrm{CP}$ can fuse with $\mathrm{TiO}_{2}$. In addition, this complex system showed higher stability that zwitterionic phosphocholine (PC) lipids $\mathrm{PC} / \mathrm{SiO}_{2}$ system, which was indicated by washing the membrane under harsh conditions. Interestingly, the CP liposome cannot fuse with 
silica surface because of a strong charge repulsion.

This platform made the foundation for further investigations on combining inorganic materials with liposomes. The composite nanoparticles containing silica core and gold shell exhibit huge potential in treating cancer by photodynamic therapy. However, these nanoparticles show limited ability in carrying drugs, thus Wu et al.[75] utilized the excellent drug loading capability of liposomes to carry ariamycin. Furthermore, these nanoparticles were coated by silica and then by gold. This composite system took advantages of both chemotherapy and photothermal therapy to implement combination therapy to cancer. He et al.[76] connected phospholipid material with $\mathrm{Fe}_{3} \mathrm{O}_{4} @ \mathrm{SiO}_{2}$ nanoparticles by covalent combination, then these materials were used to fabricate horseradish peroxidase-loaded liposomes. The generated liposomes can detect the presence of $\mathrm{Cu}^{2+}$ by naked eyes, providing a novel diagnostic strategy to clinical applications.

\subsection{Hydrogel combined with liposomes}

Hydrogel is a gel using water as a dispersion medium, which partly introduces some hydrophobic and hydrophilic groups to the crosslinked structure of the hydrophilic polymer, and the hydrophilic groups can interact with water molecules in the inner structure. In addition, these hydrophobic compositions can expand by combining the crosslinked polymers with water molecules. Because of the soft texture, excellent biocompatibility, and the ability in maintaining certain shape, these types of materials have been widely utilized in biomedical field. According to the source of materials, hydrogels are mainly divided into two types, synthetic polymeric hydrogels and natural polymeric hydrogels[77].

In general, synthetic polymer hydrogels are primarily consisting of polyacrylic acid, polyvinyl alcohol, polyacrylamide, etc., which have outstanding mechanical strength, excellent chemical stability, and clear composition. However, these materials still face some problems, such as poor biocompatibility and toxic 
degradation productions. Different attempts are made to make these materials as excellent candidate for clinical applications. For example, Suri et al.[78] prepared unilamellar liposomes by solvent evaporation and rehydration. Then without any heating or chemical crosslinking, these liposomes were combined with PVA to fabricate composite hydrogel with the repeated freeze thawing to induce cross-linking. During the whole process of fabrication, any heating or chemical crosslinking was avoided, which makes sure the biocompatibility of this composite system, thus this material was promising to be a candidate for biomimetic sensing and implantable tissue applications.

Kazakov et al.[79] modified N-octadecyl acrylamide on the surface of poly $\mathrm{N}$-isopropylacrylamide-co-1-vinylimidazole hydrogel nanoparticles, then these hydrogel nanoparticles were heated above the phase transition temperature of liposomes. The integration between the hydrophobic chains of hydrogels and the phospholipid component of liposomes were observed by dynamic light scattering (DLS) and atomic force microscope (AFM). These hydrogel nanoparticles were covered into the inner phase of liposomes generating complex hydrogel nanoparticles coated with the phospholipid bilayer. These nanoparticles provided a novel platform to drug delivery and biophysical nanodevices.

Mourtas et al.[80] tried to use phospholipids, hydrogenated lecithin, and cholesterol at different proportion to prepare small unilamellar vesicles and multi-compartment liposomes. Furthermore, these liposomes were mixed with carbomer 974 and hydroxyethyl cellulose to make different hydrogels, then these hydrogels were investigated by rheometer to explore their rheological properties. As shown by the results, liposome composition (membrane rigidity) and lipid concentration, but not liposome size, seem to be very important factors that determine the rheological modulations caused by liposome addition in gels. This work indicated that gel rheological properties may influence drug release kinetics, thus the implications of the liposomes on the final product rheological profile should be seriously considered. Gao et al.[81] utilized gold nanoparticles to modify the surface of liposomes, then these liposomes were further mixed with acrylamide, 
ammonium persulfate, polyethylene glycol dimethacrylate, tetramethyl ethylenediamine at the room temperature for $4 \mathrm{~h}$ to obtain the complex hydrogels. A pH-dependent fusion with bacterial membrane was observed in Staphylococcus aureus bacteria co-cultured with hydrogel formulation releasing nanoparticle-stabilized liposomes. When applying this material on the skin of Sprague Dawley (SD) rats, no significant skin toxicity was found after 7 days' treatment, which indicated that this material could be a promising anti-bacterial scaffold in local area.

Natural polymer hydrogels primarily involve collagen, gelation, HA, and CS. This type of hydrogel is famous for outstanding biocompatibility, and absorbable degradation products, which has been widely used in clinical treatment[82]. However, the mechanical strength and stability of those materials should be further improved to broaden the applications. Several efforts have been done to make those materials more suitable for clinical treatments.

Gariepy et al.[83] firstly modified chitosan- $\beta$-glycerol with liposomes to make novel hydrogels. They found that liposomes can significantly improve the viscoelasticity and mechanical strength of this complex system. Moreover, CS can noticeably prolong the release time of hydrophilic drugs from $24 \mathrm{~h}$ to over two weeks in vitro. Lu et al.[84] connected aldehyde-modified xanthan gum with amine-modified liposome by dynamic Schiff base reactions to fabricate injectable self-healing hydrogel. Furthermore, MCF7 cells were cultured in this 3D scaffolds, which indicated that this system could be an intelligent carrier for cell therapy in tissue engineering. Li et al.[85] fabricated liposomes coated with thiolated-chitosan and maleated-chitosan, respectively. Then the two types of liposomes were combined with each other by thiol-olefin reactions, generating a chitosan/liposome composite hydrogel. the gel time and swelling ratio can be regulated by changing $\mathrm{n}_{-\mathrm{SH}} / \mathrm{n}_{-\mathrm{C}=\mathrm{C}-}$ ratio. After adding liposomes, the tensile strength of the gels increased, and the crystal became smaller. After culturing with HaCaT cell line, the hydrogel showed excellent biocompatibility in vitro, which indicated that this system could be regarded as drug-loaded scaffolds for tissue engineering. O'Neill et al.[86] blended 
thermosensitive liposomes with thermosensitive chitosan hydrogels to prepare injectable thermosensitive composite chitosan hydrogels. After local injection and the stimulation of heating, the loaded drug deferoxamine was released from scaffolds to recruit stem cells and improve the expression level of VEGF, as shown in Figure 6. This responsive, local and specific release method provided a novel administration for tissue engineering.

\subsection{Electrospinning fibers combined with liposomes}

As an electrostatic stretching spinning method for polymer, first the polymer solution is incubated under static electricity at thousands or even tens thousands of volts and then under the action of electric field force, these charged droplets are accelerated at the Taylor cone apex of the capillary. When the electric field force is strong enough, these polymer droplets will overcome surface tension and form jetting stream. During the process of jetting, the solvent is evaporated, and the solidified stream is collected to obtain electrospinning fibers[87]. The diameters of electrospinning fibers are usually smaller than the size of cells, and in this case, these fibers can mimic the structure and biological function of natural extracellular matrix[88]. Most of human tissues and organs have similar form and structure of nanofibers, which makes the electrospinning fibers be popular in tissue engineering. Some polymer materials have outstanding biocompatibility and degradability, which could be regarded as favorable scaffolds. Moreover, electrospinning fibers own large specific surface areas and porosity, providing a suitable platform for further modifying or applications[89].

The electrospinning fibers are also often modified by liposomes, from surface modification, internal loading to self-assembly. For example, pre-electrospinning solvent was prepared by mixing liposomes solution with polymer solution, then electrospinning technology was utilized to fabricate composite fibers loaded with liposomes. The fibers can significantly prolong the drug release in liposomes. For examples, Li et al.[90] prepared naproxen-loaded liposomes with sodium 
hyaluronate as stabilizer, then coaxial electrospinning technology was performed to fabricate fibers with core-shell structure. Liposomes were loaded in the core part to prolong the drug release period to up to two weeks. This material used as wound dressing could release analgesic for period in local area, which makes the treatment more efficiently.

Lin et al. [91] mosaiced proteins into the membrane of liposomes to fabricate cinnamon essential oil/ $\beta$-cyclodextrin loaded protein liposomes, then these liposomes were blended with polyethylene oxide to prepare novel fibers by electrospinning technology, as shown in the Figure 7. Because of the proteolysis, this fiber can release drug to kill certain bacillus cereus, providing a novel strategy to design an anti-bacterial material. Chandrawati et al.[92] prepared $\beta$-glucuronidase loaded liposomes, then they fabricated complex scaffolds by modifying PVA with these liposomes, the vitality of glucuronidase could be maintained for seven weeks. Moreover, prodrug SN-38-glucuronide could be successfully turned into antiproliferative drug $\mathrm{SN}-38$ by this scaffold. After that, antiproliferative effect could be found in the human cervical cancer cells (HeLa) treated with biocatalytic electrospun fibers. The assembled biocatalytic materials successfully produced antiproliferative drugs, as is achieved by the current most successful cardiovascular stents on the market, and effectively suppressed proliferation of cells. Therefore, this platform could be a new idea to fabricate cardiovascular grafts.

Liposomes with special functionality were grafted on the surface of electrospinning fibers though covalent bonds or non-covalent bonds. In this way, the drug is loaded in the liposomes instead of the scaffold, thus avoid the potential damage brought by high voltage electrostatic field during electrospinning. Monteiro et al.[93] processed the surface of CS fibers with some thiolation reagents, then gentamicin-loaded maleimide liposomes were grafted on the surface of fibers by covalent reactions, as shown in the Figure 8. In vitro experiments indicated that gentamicin released from the liposomes immobilized at the surface of electrospinning fibers has antibacterial activity against Escherichia coli, Pseudomonas aeruginosa and Staphylococcus aureus. Therefore, these results show that the 
developed nanostructured delivery system has promising performance for wound dressing applications, and could be used in the eradication of these pathogens, which are a common cause of local infections Furthermore, they utilized thiolation reagents to treat the surface of polycaprolactone $(P C L)$, then the dexamethasone-loaded liposomes were grafted on the surface of these PCL fibers through generating covalent bonds between maleimide and thiol. This scaffold could constantly release dexamethasone in the local area, promoting the osteogenic differentiation of human mesenchymal stem cells, which could be regarded as advanced drug carriers for controlling growth factors release. Batory et al.[94] fabricated nanogold, nanosilver and nanocopper-loaded liposomes. Then these liposomes were grafted on the surface of PCL fibers to produce composite fibers with the capability in anti-bacterial, antifungal, promoting the creation of collagen and elastin.

Another type of popular methods to fabricate composite fibers with liposomes is blending lipid materials with water-soluble polymer. When these composite fibers were dissolved in water, liposomes would be generated by self-assembly. Recently, this method has attracted wide attention from researchers, for example, polyvinylpyrrolidone (PVP) $\mathrm{K} 60$ and lecithin were utilized by $\mathrm{Yu}$ et al.[95] to make amphiphilic nanofibers. When these fibers were immersed into water, phosphatidylcholine liposome could be generated by self-assembly. Moreover, the diameter of liposomes could be controlled by regulating the proportion of lecithin. Liposomes generated by this method can provide strategies for developing multi-functional and novel drug carriers.

\subsection{Summary}

Although liposomes are usually as a liquid formulation, it could be combined with biomaterials in diverse types, such as hydrogel and electrospinning fibers to fabricate multifunctional composite systems. Associating liposomes with hydrogel can make composite system constantly release drug in the local area and supply efficiently mechanical support. Moreover, this hydrogel can also provide favorable 
microenvironment to the cells in the local area. Combining liposomes with different types of electrospinning fibers provides some novel strategies to fabricate self-assembly drug delivery systems and also staged drug release systems. Therefore, the composite systems created by combining liposomes with various types of biomaterials are considered to be worth to translate into clinical applications.

\section{The application of scaffolds combined with liposomes in disease treatment}

In 1988, Swiss Cilag registered the econazole liposome gel to treat the skin diseases, which was the first liposome-based product, and now, this gel is sold in Switzerland, Italy, Belgium and other countries[96]. After that, various kinds of liposomes-based products were proposed, such as nystatin liposomes[97], hepatitis A vaccine liposomes[98], and cytarabine liposomes[99]. Some anti-cancer drug-loaded liposomes, anti-infection drug loaded liposomes and gene drug-loaded liposomes are under clinical trials. In addition, liposomes also exhibit promising characteristics in overcoming tumor resistance and biological barriers. Overall, these achievements in liposomes make the foundation for developing intelligent multifunctional liposomes-scaffold composed systems in treating various types of diseases, as shown in Table 2.

\subsection{Cancer therapy}

Most of liposomes used for cancer therapy consist of the derivative of 1,2-distearoyl-sn-glycero-3-phosphoethanolamine- (polyethylene glycol) (DSPE-PEG). These materials can prevent liposomes from phagocytosis by mononuclear macrophage, leading to the prolonged circulation time and the increased accumulation in lesion. Thus, these liposomes are named as long-circulation liposomes. In cancer patient, the permeability of capillaries in lesion is increased, because of the inflammation and infection caused by the growth of solid tumor. 
Therefore, nanoparticles with suitable diameter can go through capillaries and accumulate in the lesion, which is also known as passive targeting ability of liposomes. After some specific surface modifications like antibodies, hormones, sugars residue, receptor ligands, liposomes can show active cancer targeting ability, and additionally, liposomes can be designed to exhibit responses to some cancer relative or outside applied stimulations, such as $\mathrm{pH}$, temperature, and magnetic field. Certain liposomes can also bring drugs to targeted organs, cells, or even subcellular organelles. All these characteristics of liposomes make them promised for designing composite liposomes scaffolds in cancer therapy.

Mao et al. [100] prepared a thermosensitive injectable hydrogel containing paclitaxel-loaded liposomes, then the transform temperature, diameter, and drug release rate were investigated in vitro. Furthermore, the system was evaluated in a pancreatic cancer mice model and constant drug release and improved drug concentration was observed in the local area. Moreover, this system did not exhibit any significant toxicity to surrounding normal tissues.

In another work, Xing et al.[101] combined 2-methoxyestradiol loaded liposomes with injectable thermosensitive PLGA-PEG-PLGA polymer to fabricate hydrogel with two-stage controlled drug release. In vivo experiments indicated that these liposomes could be transported to the local tumor area, improving the chemotherapy efficiency and bioavailability of the drug. In normal physiological conditions, 7-ethyl-10-hydroxycamptothecin (SN-38) could easily transform between active lactone form (SN-38A) to inactive carboxylic acid ester form (SN-38I) due to its low solubility. To solve this problem, Bai et al.[102] prepared acid SN-38 liposomes loaded in thermosensitive hydrogel, and after that, a series of experiments were carried out to explore the anticancer efficiency and biocompatibility of this composite systems. Usually, compound drug delivery systems contain organic nanocarriers and inorganic nanocarriers, and at the same time is expected to achieve its complementary advantages for the aim of improving the performance of antineoplastic drugs in cancer tumor therapy.

Zhang et al.[103] modified gold nanoparticles with paclitaxel through covalent 
bonds, and then the products were inserted into the bilayer of liposomes to construct a complex nanosystem. This system was utilized to treat mouse with liver cancer, and the pharmacokinetics, biodistribution, and anticancer efficiency were evaluated. The results indicated that integrated hybrid liposome had superior properties, including improved stability of the whole system, enhanced cellular uptake, and rapid-and-then-sustained drug release, resulting in enhanced tumor cell killing ability in vitro and tumor therapeutic efficacy in vivo.

\subsection{Skin-related diseases}

Skin is the biggest organ in human body, which consists of epidermis, dermis and subcutaneous tissue. The main functions of skin are to protect the body, feel temperature and pressure, and it can also protect various types of tissues and organs in body from the invasion created by pathogenic microorganism [104]. According to the features of skin, some researches try to fabricate some composite systems, which could meet the clinical demands. For example, paeonol is usually used to treat some skin-related disease, because it shows potential abilities in analgesia, anti-inflammatory, antipyretic and anti-allergic reactions[105]. But this drug has unsatisfactory solubility. Hydrogel is regarded as an ideal delivery carrier in the local area. To improve the solubility of paeonol, Xia et al.[106] fabricated paeonol-loaded liposomes. Then these liposomes were combined with hydrogel to enhance the local retention time and the affinity with the skin for achieving a better treatment effect.

Adapalene (ADA) is the second generation of retinoic acid, exhibiting certain treatment effect in diverse skin diseases, such as acne vulgaris; however, this drug has dose-dependent side effects. To make this drug more suitable for clinical application, Jain et al. [107] prepared ADA-contained solid lipid nanoparticles, further these particles were combined with carbomer hydrogel to fabricate composite system. Then the drug release, biodistribution, skin penetration and rheology were investigated. The results indicated that this system can prevent ADA from systematically absorbing by skin, and release drug in local area, providing a 
novel strategy to treat hemorrhoids. Mandlik et al.[108] fabricated sertaconazole nitrate loaded liposomes with the diameter around $246.2 \pm 2.49 \mathrm{~nm}$ and encapsulation efficiency of $86.16 \pm 0.56 \%$ by orthogonal test. Furthermore, these nanoparticles were blended with hydrogel. In this composite system, the percentage of drug spread was $13.24 \%$, and the deposition rate of drug in skin was $83.54 \%$. In vitro, antibacterial ring experiment indicated that the ring of this hydrogel was 33 $\mathrm{mm}$, which was significantly higher than $22 \mathrm{~mm}$ of the commercial materials, meaning that this novel material has stronger anti-bacterial activity.

\subsection{Diabetes}

Type I diabetes, also known as insulin dependent diabetes, is one of the most popular disease in the world. To treat this disease effectively, Haque et al.[109] prepared a kind of injectable hydrogel containing isolated pancreatic islets and clodronate loaded liposomes at the same time, and then this hydrogel was utilized to treat SD rats without pancreatic islets. The results indicated that the hydrogel including isolated pancreatic islets and clodronate liposome, significantly enhanced the median survival time of SD rats for over 60 days. Furthermore, to solve a series of side effects, such as suffering brought by repeated injections, infection caused by wearing an insulin pump in catheter area, Chen et al.[110] developed a thermosensitive hydrogel modified with insulin-loaded liposomes to minimize the time of injection. By utilizing the theory that thermosensitive hydrogel could be liquid under low temperature and turn into gel at $37^{\circ} \mathrm{C}$, hydrogel was formed in situ to constantly release insulin for 7 days to provide a strategy for diabetes treatment.

\subsection{Anti-inflammatory diseases}

Inflammation is part of the complex biological response of body tissues to harmful stimuli, such as pathogens, damaged cells, or irritants, and is a protective response involving immune cells, blood vessels, and molecular mediators. Additionally, the 
main symptoms of inflammation are red, swelling, fever, pain and disfunction[111]. In the process of inflammation, on one hand, the endogenous damaging factors directly or indirectly destroy tissues and cells, and on the other hand, the factors are diluted and inactivated by exudation and congestion caused by inflammation[112]. At the same time, the regeneration of parenchymal cells and interstitial cells leads to tissue repair and healing, therefore inflammation is considered as an incorporate process, including both damage and anti-damage. Scaffolds combined with liposomes have also been used for anti-inflammatory.

For example, Negi et al.[113] fabricated resveratrol-loaded liposomes by taking advantage of both ethanol injection and film dispersion method, then these liposomes were further processed with a hydrogel. The generated hydrogel system was confirmed to follow Korsmeyer-Peppas model in different drug release condition in vitro. Especially, in the model of swelling mouse paw induced by carrageenan, this system could significantly prolong the treatment time and reduce the extent of swelling in vitro, compared with standard diclofenac sodium gel. Furthermore, resveratrol also showed higher permeability and deposition in skin.

\subsection{Human immunodeficiency virus (HIV)}

HIV is a virus that can cause defect in human immune system and mainly attack human helper T lymphocyte system. Once, this virus irrupts human cells, it will integrate with the normal cells, thus it is extremely difficult to eliminate this virus. Moreover, this virus infection induced high mortality rate and long incubation period [114]. A composite system was fabricated by Ramanathan et al.[115] using hydrogel as a core structure and a phospholipid bilayer as shell structure to carry maraviroc and tenofovir disoproxil fumarate. This system was applied in the treatment of HIV in mouse by absorbing in mouse vaginal mucosa, leading to antiviral activity against HIV-1 BaL in cervicovaginal lavages.

\subsection{Anti-bacterial applications}


Bacterial infection leads to a series of symptoms in patients like long lasting fever, headache, nausea, and vomiting [116]. Thus, it is meaningful to investigate anti-bacterial treatment in order to get better recovery in patients. Specifically, chitosan hydrogel was combined with mupirocin-loaded liposomes to build complex hydrogel system, then the biocompatibility and anti-bacterial capability of this hydrogel were explored in vitro, and mouse burn model was utilized to evaluate the potential ability of hydrogel in vivo. As shown by the results, the delivery system enhanced wound healing and was equally potent as the marketed product of mupirocin[117].

\subsection{Summary}

Advanced scaffolds modified with liposomes were applied in various types of areas, such as tumor, skin disease, diabetes, inflammation, HIV and infection. Moreover, these applications exhibit some benefits due to special properties of scaffolds, such as mechanical supporting, 3D structure and therapy functions brought by drug-loaded liposomes, such as anti-cancer, antibacterial, and anti-inflammation. However, these materials also meet some challenges in application, for example, some local inflammation could be caused by implanted scaffolds; under the physical conditions, the drug release behavior could be different from the lab testing. Making the properties of composite scaffolds closer to the original tissues or organs could be a promising strategy for these materials.

\section{The application of scaffolds modified with liposomes in tissue regeneration}

\subsection{Bone regeneration}

During bone regeneration there is stimulation of various kind of cytokines, osteogenic precursor cells, including osteoblasts and osteoclasts are activated. 
Firstly, these cells are involved in the process of bone resorption, specifically osteogenic precursor cells are attracted to the site of bone resorption, then proliferation of pre-osteoblasts can be observed under the effect of diverse cytokines. Furthermore, these pre-osteoblasts differentiate to mature osteoblasts that can combine with osteogenic proteins, including type I collagen, osteocalcin, alkaline phosphatase, glycoprotein, and growth factors to generate matrix mineralization. Because of the effect of cytokines, some morphological changes happen in osteoblasts, leading to the drop of these cells from the surface of bone, then the circulating osteoclasts can attach through the bone surface, triggering the process of bone resorption. During this process, the osteoblasts surrounding osteoclasts also take part in bone resorption by decomposing the collagen in the bone lacuna. After that, the remained components and generated cytokines can act on osteoclasts to induce the bone generation. Many types of cytokines take part in regulating the process of bone regeneration[118]. In addition, the mechanical properties of the whole system can be influenced by bone defect, because the lack of mechanical supporting and $3 \mathrm{D}$ environment would cause the decrease in cell proliferation, attachment and differentiation in the local defect area[119]. Therefore, composite scaffolds, which can release drug in situ for long-term and fill the defect area will provide certain mechanical support for the entire system, have promising prospect in clinical applications.

The nanofibers structure of bone proteins was mimicked by poly PLA nanofibers (by Mohammadi et al.[120]), then these fibers were coated with hydroxyapatite nanoparticles to simulate bone mineralization. Furthermore, bone morphogenetic protein-2 peptide loaded liposomes were grafted on the scaffolds by covalent bond to regulate the release rate of peptides. As shown in Figure 9 favorable biocompatibility and satisfactory ability in promoting the osteogenic differentiation of MSC can be observed in this scaffold. In another work, non-phospholipid liposome was fabricated by Cui et al.[121] by using bipolar single-chain molecule with high sterol content. Moreover, hydroxycholesterol was proved to have the capability in stimulating bone formation, thus 20(S)-hydroxycholesterol was used as a 
composition in the liposomes. Furthermore, the cytocompatibility and osteogenic capacity of this liposomes were proved by combining this nanomaterial with hydrogel in vitro, which provides a novel administration strategy that non-phospholipid liposome is regarded as a platform for delivering small drug molecules or genes to promote bone regeneration. Deferoxamine (DFO) is a small molecule hydrophilic drug that can upregulate the expression of hypoxia-inducible factor 1- $\alpha)($ HIF-1 $\alpha)$ and VEGF in bone and serum to enhance the angiogenesis in bone. Additionally, the high expression of osteogenic related proteins in the Wnt signal pathway further accelerating the bone formation. However, because of high hydrophilicity, it is challenging for this drug to maintain effective concentration in the local area. Thus, Chen et al.[122] fabricated DFO-loaded liposomes and modified gelatin methacryloyl (GelMA) with these liposomes to release drug in situ for long-term, promoting the angiogenesis and bone regeneration.

\subsection{Wound healing}

Wound healing is a recovery process after defect or disconnect occurs in skin or other related tissues. This process mainly includes the regeneration of various tissue (including epidermis and dermis), the proliferation of granulation tissue and the formation of scar tissue, where diverse synergy effects can be found in this process[123]. At the same time, angiogenesis, inflammation and anti-infection play important roles in wound healing.

Using traditional anti-infection methods to treat partial thickness burns cannot provide humid environment to promote wound healing. Therefore, Homann et al. [124] took advantage of liposomes-modified polyvinylpyrrolidone-iodine hydrogel to treat 43 randomly selected patients with second degree burn wound. The patients treated with commercial sulfa silver ointment were regarded as control groups. The results indicated that patients handled with hydrogel had better prognostic, because higher water binding rate was found in the composite hydrogel system, which could provide necessary humidity to the defect area. Moreover, it was much easier to 
change the hydrogel dressing, which caused less injury on the burn wound.

Fibroblast growth factors (FGF) play vital roles in the routing of the wound healing, because they show ability in promoting the migration of endothelial cells and the proliferation of smooth muscle cells. Additionally, it can also accelerate the formation of new blood vessel and repair damaged endothelial cells. Therefore, how to maintain the effective concentration of FGF on the wound area with lots of wound leachate is a key factor in the process of treating wounds. Basic fibroblast growth factors (bFGF) loaded-silk fibroin hydrogel was embed into the inner phase in liposomes by Xu et al.[125], and then superior capability in controlling release of bFGF and also promoting bFGF penetration was observed in this complex system. This platform could be regarded as an excellent carrier to deliver growth factors to the wound area.

Wound, especially burn wound, tends to cause life threatens to patients, because of infection. But, the risk of wound infection can be decreased in a long-term by administrating enough antibiotic in the local wound area. Specifically, mupirocin was utilized as a model drug by Hurler et al.[126] to make drug-loaded liposomes. Then composite system was fabricated by mixing these liposomes with chitosan hydrogel, which exhibited significant longer duration of drug release both in vivo and in vitro. Additionally, drug release rate was regulated by controlling the diameter of liposomes. Noticeable inhibition effect on staphylococcus aureus and bacillus subtilis was observed. Moreover, comparing with commercial mupirocin cream, this system showed more powerful capability in antibacterial and bio-adhesion.

\subsection{Spinal cord repairment}

Permanent functional defect will be caused by spinal cord injury, including neuronal and axonal damage; moreover, the regeneration ability of spinal cord is limited, therefore it is still a big challenge to treat spinal cord injury in clinic [127]. Recently, neural stem cells (NSCs) are gradually regarded as cellular sources for spinal cord 
regeneration, because those cells exhibit potential ability in differentiating into neurons, astrocytes and oligodendrocytes, and then establishing connection with host cells [128]. However, because of the limits brought by local microenvironment, most of these neural stem cells tend to differentiate into astrocytes or oligodendrocytes instead of neurons. Therefore, it is meaningful to induce these neural stem cells to differentiate into neurons for spinal cord regeneration.

According to reports, after spinal cord injury, microtubule-stabilizing agent paclitaxel (MSPTX) could reduce the generation of scar and enhance the regeneration of inner axon. Thus, a composite collagen microwell scaffold containing MSPTX-loaded liposomes and neural stem cells was fabricated by Li et al.[129]. The PTX functionalized collagen scaffolds could not only alleviate myelin inhibition, but also enhance intrinsic neuronal differentiation potential of NSCs in vitro. Furthermore, this scaffold was implanted into the T8 transverse part of mouse spinal cord, as shown in Figure 10. The functional scaffolds could provide an instructive microenvironment for NSCs to differentiate into mature neurons and functional sensory and motor neurons. Together, this scaffold provided a suitable microenvironment for the differentiation of neural stem cells, the regeneration of motor neurons and sensory neurons, the attendance of axon, making improvements in the motor evoked potentials and hindlimb movement.

\subsection{Teeth regeneration}

Currently, the life spans of dental restorations are relatively short. Therefore, utilizing tissue engineering to promote the regeneration of dentin pulp complex means a lot to the successful implant of dental restorations. There are plenty of bioactive factors and extracellular matrix proteins that are related with the recruitment, proliferation and differentiation of pulp progenitor cells in dentin matrix. Therefore, Melling et al.[130] fabricated decalcified dental matrix loaded liposomes (DDM-Lip) to repair the teeth tissues. As the results showed, more efficiency in recruiting and activating dental pulp stem cell (DPSC) could be found in 
samples treated with DDM-Lip, comparing to samples treated with DDM. Moreover, the recruitment and activity of DPSC are necessary factors in the process of teeth tissues regeneration. This research proved the potential capability of DDM-Lip in stimulating teeth regeneration in vitro, which supply a novel strategy for teeth repair and hard tissue engineering.

\subsection{Summary}

The smart composite scaffolds modified with liposomes are widely utilized in the tissue regeneration, because of its favorable biocompatibility, and controlled drug release in situ, as shown in Figure 3. Moreover, it can also provide sufficiently mechanical support to the defect area and suitable microenvironment for the cells' adhesion and proliferation. During the process of tissue regeneration, regulating cytokines and promoting differentiation of cells play important roles. Thus, to broaden the application of composite scaffolds in tissue regeneration, some modification could be processed in lecithin, which is the major composition for liposomes. For example, taking advantages of both liposomes and proteins from the cell membrane, different types of cytokine-loaded liposomes could be modified with diverse cell membrane, and further combined with scaffold to construct novel composite implant, which is more suitable for clinical applications.

\section{Conclusion and further perspectives}

Liposomes are nanocarriers that can maintain drugs in the lipid bilayer and inner core. The inner part of the carriers can be used to load some hydrophilic drugs, such as small molecules or macromolecules like proteins, antibodies, and cytokines. At the same time, some lipid drugs can be carried on the bilayer to achieve the goal of co-delivery drugs with different characteristics. As drug carriers, liposomes exhibit many advantages, such as lymphatic system tropism, passive targeting on bone marrow and spleen, excellent ability in controlling drug release, reducing drugs side 
effects, and improving the stability of drug. However, liposomes still face some challenges, for example, the liposomes have some troubles in transporting and store drugs. Moreover, it is difficult for liposomes to maintain certain drug concentrations in the local area for long periods of time. Therefore, to make liposomes more suitable for the clinical applications, these disadvantages should be solved without compromising their advantage properties.

Scaffolds have attracted wide attention from the researchers, because they can be used in diagnosis, repairment or enhancement of human tissues or organs. Moreover, they own unique functions that cannot be replaced by drugs. Although, certain mechanical supporting and suitable microenvironment for proliferation can be supplied by scaffolds in situ and scaffolds can control the flow of blood and body fluid, their ability to recover the defect is weak. Thus, further functionalization is needed for scaffolds in order to broaden their applications in clinic.

Fabricating composite scaffolds combined with liposomes cannot only provide scaffolds with the ability to promote regeneration and treating diseases, but also maintain the drug concentration in situ for a long period of time. Additionally, scaffolds modified with liposomes showed sustained drug release. Furthermore, uniform liposome solution with different types of drugs can be easily formed, which can be used to distribute drugs evenly in the scaffold. By this method, liposome composite scaffolds can deliver diverse medicines and achieve the goal of synergistic therapy.

Favorable combination between liposomes and scaffolds can be obtained by adjusting the composition of the phospholipid bilayer to avoid the generation of interface separation. Moreover, significantly mechanical enhancement of the composite scaffolds can be achieved by regulating the covalent bonds or non-covalent bonds between liposomes and scaffolds.

Currently, a series of achievements have been obtained by this composite system, for example, in the tumor resection model, the continued drug release shows an inhibition effect on residual tumor. Additionally, the implant supplies suitable microenvironment for the cells' adhesion and proliferation so that 
satisfactory effects in anti-tumor and promoting tissue regeneration are observed. However, for the composite system, there are still some challenges, for example, when the drug is delivered by this method, it will be difficult to calculate and give the specific dosage to the patients or experimental animal. Because the drug is firstly loaded into liposomes, then the drug-loaded liposomes are further combined with different scaffolds, during the whole process the loaded-drug can leak out of the system. In the bone defect area, scaffolds can provide certain mechanical supporting, and at the same time, liposomes can accelerate the osteogenic differentiation by controlling drug release in situ. Some potential issues can cause problems in this application, for example, the degradation period of scaffold cannot match with the regeneration rate of bone, then the newborn bone will cover the scaffold, finally the remained implants could hinder the bone regeneration. In the wound healing applications, dressings are usually regarded as an anti-infecting physical barrier, which can provide suitable humidity and regeneration environment to the wound, meanwhile, the continuously released antibacterial drugs can play an anti-infective role. Sometimes, the infected area consists of not one type bacterium, but different kinds of bacteria Thus, if the loaded drug cannot meet the demand to clear all these bacteria, then the surviving bacterium can be resistant to the following treatment.

Based on some current novel researches, the following liposome-scaffold system can be the future research trend. Specifically, (1) liposome-scaffold, including diagnosis, imaging and therapy in one composite system; (2). liposomes fused with different kind of cell membranes, according to the clinical demands, then combine with scaffolds to treat diseases and accelerate tissues repairment by taking the advantage of the immune therapy and tissues engineering; and (3) self-assembly drug delivery systems and staged responsive delivery systems fabricated by combining scaffolds and liposomes would provide promising prospects to disease treatment and tissue regeneration. 


\section{Disclosure statement}

The authors declare no competing financial interest.

\section{Acknowledgements}

This work was supported by the National Natural Science Foundation of China (51873107 and 81871472), Shanghai Municipal Education Commission-Gaofeng Clinical Medicine Grant Support (20171906), Shanghai talent development fund (2018099). Prof. H. Z. acknowledges financial support from Academy of Finland (grant no. 297580) and Sigrid Jusélius Foundation (decision no. 28001830K1). Prof. H. A. S. acknowledges financial support from the HiLIFE Research Funds and the Sigrid Jusélius Foundation.

\section{Reference}

[1] A. Gabizon, R. Shiota, D.. Papahadjopoulos, Pharmacokinetics and tissue distribution of doxorubicin encapsulated in stable liposomes with long circulation times, J Natl Cancer I, 81(19) (1989) 1484-8.

[2] A. Samad, Y. Sultana, M. Aqil, Liposomal drug delivery systems: an update review, Curr Drug Deliv 4(4) (2007) 297-305

[3] R. Langer, J. Vacanti, Tissue Engineering, Mol Ther. 1(1) (2000) 12-15.

[4] D.W.J.B. Hutmacher, Scaffolds in tissue engineering bone and cartilage, Biomaterials 21(24) (2000) 2529-2543.

[5] A.D. Bangham, Liposomes: the Babraham connection, Chem Phys Lipids 64(1-3) (1993) 275.

[6] T. Lian, R.J. Ho, Trends and developments in liposome drug delivery systems, J Pharm Sci-US 90(6) (2001) 667-680.

[7] T.S. Levchenko, W.C. Hartner, V.P. Torchilin, Liposomes in diagnosis and treatment of cardiovascular disorders, Methodist Debakey Cardiovasc J 8(1) (2012) 36-41.

[8] M.S. Martina, J.P. Fortin, C. Ménager, O. Clément, G. Barratt, C. Grabielle-Madelmont, F. Gazeau, V. Cabuil, S.J. Lesieur, Generation of superparamagnetic liposomes revealed as highly efficient MRI contrast agents for in vivo imaging, J Am Chem Soc. 127(30) (2005) 10676-10685.

[9] L.G. de La Torre, S.C. de Pinho, Lipid matrices for nanoencapsulation in food: liposomes and lipid nanoparticles, Food nanoscience and nanotechnology, (2015), pp. 99-143. 
[10] N. Moussaoui, M. Cansell, A.J. Denizot, Marinosomes ${ }^{\circledR}$, marine lipid-based liposomes: physical characterization and potential application in cosmetics, Int J Pharm 242(1-2) (2002) 361-365.

[11] R.A. Schwendener, Liposomes in biology and medicine, Bio Appl Nanoparticles, (2007), pp. 117-128.

[12] F. Kong, X. Zhang, H. Zhang, X. Qu, D. Chen, M. Servos, E. Mäkilä, J. Salonen, H.A. Santos, M.J.A.F.M. Hai, Inhibition of Multidrug Resistance of Cancer Cells by Co-Delivery of DNA Nanostructures and Drugs Using Porous Silicon Nanoparticles@ Giant Liposomes, Adv. Funct. Mater. 25(22) (2015) 3330-3340.

[13] D. Carugo, E. Bottaro, J. Owen, E. Stride, C.J.S.r. Nastruzzi, Liposome production by microfluidics: potential and limiting factors, Sci. Rep-UK 6 (2016) 25876.

[14] I. Takeuchi, N. Kishi, K. Shiokawa, H. Uchiro, K. Makino, Polyborane encapsulated liposomes prepared using $\mathrm{pH}$ gradient and reverse-phase evaporation for boron neutron capture therapy: biodistribution in tumor-bearing mice, Colloid Polym Sci. 296(7) (2018) 1137-1144

[15] X. Fu, Y. Lu, J. Guo, H. Liu, A. Deng, C. Kuang, X. Xie, K237-modified thermosensitive liposome enhanced the delivery efficiency and cytotoxicity of paclitaxel, J Liposome Res. 29(1) (2019) 1-8.

[16] C. Liao, X.D. Qiao, L.X. Hong, F.Y. Qi, Y. Jun, L.X. Fang, G.B. Hong, Iridium (III) complex-loaded liposomes as a drug delivery system for lung cancer through mitochondrial dysfunction, Int J Nanomedicine. 13 (2018) 4417-4431.

[17] Y. Zhao, A. Liu, Y. Du, Y. Cao, S. Zhang, Effects of Sucrose Ester Structures on Liposome-Mediated Gene Delivery, Acta Biomater. 72 (2018) 278-286.

[18] Y.Z. Zhao, D.L. Zhu Ge, M.Q. Tong, M.T. Lin, Y.W. Zheng, X. Jiang, W.G. Yang, Q. Yao, Q. Xiang, X.K. Li, H.L. Xu, Ulcerative colitis-specific delivery of keratinocyte growth factor by neutrophils-simulated liposomes facilitates the morphologic and functional recovery of the damaged colon through alleviating the inflammation, J Control Release 299 (2019) 90-106.

[19] G. Dapergolas, G.J.L. Gregoriadis, Hypoglycaemic effect of liposome-entrapped insulin administered intragastrically into rats. Lancet, 2(7990) (1976) 824.

[20] Y. Yamada, L. Burger, E. Kawamura, H.J.B. Harashima, P. Bulletin, Packaging of the Coenzyme Q10 into a Liposome for Mitochondrial Delivery and the Intracellular Observation in Patient Derived Mitochondrial Disease Cells, 40(12) (2017) 2183-2190.

[21] Y. Doi, T. Shimizu, Y. Ishima, T. Ishida, Long-term storage of PEGylated liposomal oxaliplatin with improved stability and long circulation times in vivo, Int J Pharm. 564 (2019). 237-243

[22] L. Willerding, S. Limmer, M. Hossann, A. Zengerle, K. Wachholz, T.L.M.T. Hagen, G.A. Koning, R. Sroka, L.H. Lindner, M. Peller, Method of hyperthermia and tumor size influence effectiveness of doxorubicin release from thermosensitive liposomes in experimental tumors, J Control Release 222(2) (2016) 47-55.

[23] Y.C. Ou, J.A. Webb, S. Faley, D. Shae, E.M. Talbert, S. Lin, C.C. Cutright, J.T. Wilson, L.M. Bellan, R.J.A.O. Bardhan, Gold Nanoantenna-Mediated Photothermal Drug Delivery from Thermosensitive Liposomes in Breast Cancer, ACS Omega, 1(2) 
(2016) 234.

[24] Y. Zhao, W. Ren, T. Zhong, S. Zhang, D. Huang, Y. Guo, X. Yao, C. Wang, W.Q. Zhang, X. Zhang, Q. Zhang, Tumor-specific pH-responsive peptide-modified $\mathrm{pH}$-sensitive liposomes containing doxorubicin for enhancing glioma targeting and anti-tumor activity, J Control Release 222 (2016) 56-66.

[25] Q. Chen, H. Ding, J. Zhou, X. Zhao, J. Zhang, C. Yang, K. Li, M. Qiao, H. Hu, P.T. Ding, X.L. Zhao. Novel glycyrrhetinic acid conjugated $\mathrm{pH}$-sensitive liposomes for the delivery of doxorubicin and its antitumor activities, Rsc. Adv. 6(22) (2016) 17782-17791.

[26] X. Tian, L. Zhang, M. Yang, L. Bai, Y. Dai, Z. Yu, Y.J. Pan, Functional magnetic hybrid nanomaterials for biomedical diagnosis and treatment, Nanobiotechnology. 10(1) (2018) e1476.

[27] G.I. Béalle, R. Di Corato, J. Kolosnjaj-Tabi, V. Dupuis, O. Clément, F. Gazeau, C. Wilhelm, C.J.L. Ménager, Ultra magnetic liposomes for MR imaging, targeting, and hyperthermia, Langmuir. 28(32) (2012) 11834-11842.

[28] V.D. Nguyen, S. Zheng, J. Han, V.H. Le, J.O. Park, S.J.C. Park, S.B. Biointerfaces, Nanohybrid magnetic liposome functionalized with hyaluronic acid for enhanced cellular uptake and near-infrared-triggered drug release, Colloids surf B Biointerfaces. 154 (2017) 104-114.

[29] Y. Kono, T. Nakai, H. Taguchi, T.J.D.D. Fujita, Development of magnetic anionic liposome/atelocollagen complexes for efficient magnetic drug targeting, Durg. Deliv. 24(1) (2017) 1740-1749.

[30] A.R. Nikpoor, J. Tavakkolafshari, K. Sadri, S.A. Jalali, M.R. Jaafari, Improved tumor accumulation and therapeutic efficacy of CTLA-4-blocking antibody using liposome-encapsulated antibody: In vitro and in vivo studies, Nanomedicine. 13(8) (2017) 2671.

[31] H. Deng, K. Song, X. Zhao, Y. Li, F. Wang, J. Zhang, A. Dong, Z. Qin, Interfaces, Tumor Microenvironment Activated Membrane Fusogenic Liposome with Speedy Antibody and Doxorubicin Delivery for Synergistic Treatment of Metastatic Tumors, ACS Appl Mater Interfaces 9(11) (2017) 9315- 9326.

[32] V. Grill, M.A. Sandrucci, L.R. Di, M. Cadenaro, P. Narducci, R. Bareggi, A.M. Martelli, Biocompatibility evaluation of dental metal alloys in vitro: expression of extracellular matrix molecules and its relationship to cell proliferation rates, J Biomed Mater Res. 52(3) (2015) 479-487.

[33] Y. Okuzu, S. Fujibayashi, S. Yamaguchi, K. Yamamoto, T. Shimizu, T. Sono, K. Goto, B. Otsuki, T. Matsushita, T.J.A.B. Kokubo, Strontium and magnesium ions released from bioactive titanium metal promote early bone bonding in a rabbit implant model, Acta Biomater. 63 (2017). 383-392

[34] M. Moravej, D.J. Mantovani, Biodegradable Metals for Cardiovascular Stent Application: Interests and New Opportunities, Int J Mol Sci. 12(7) (2011) 4250-70.

[35] B.J. O'Brien, J.S. Stinson, S.R. Larsen, M.J. Eppihimer, W.M. Carroll, A platinumchromium steel for cardiovascular stents, Biomaterials. 31(14) (2010) 3755-3761.

[36] N. Ali, Y. Kousar, T.I. Okpalugo, V. Singh, M. Pease, A.A. Ogwu, J. Gracio, E. Titus, E.I. Meletis, M.J. Jackson, Human micro-vascular endothelial cell seeding on Cr-DLC 
thin films for mechanical heart valve applications, Thin solid films. 515(1) (2006) 59-65.

[37] G. Voggenreiter, S. Leiting, H. Brauer, P. Leiting, M. Majetschak, M. Bardenheuer, U.J.B. Obertacke, Immuno-inflammatory tissue reaction to stainless-steel and titanium plates used for internal fixation of long bones, Biomaterials. 24(2) (2003) 247-254.

[38] P. Zhang, H. Chen, L. Zhang, D.J.. Zhang, Anti-adhesion effects of liquid-infused textured surfaces on high-temperature stainless steel for soft tissue, Appl. Surf. Sci. 385 (2016) 249-256.

[39] M. Ogawa, Y. Tohma, H. Ohgushi, Y. Takakura, Y.J. Tanaka, Early Fixation of Cobalt-Chromium Based Alloy Surgical Implants to Bone Using a Tissue-engineering Approach, Int J Mol Sci. 13(5) (2012) 5528-5541.

[40] Y. Meng, L. Xue, Z. Li, C. Liu, Z. Jin, J. Wang, Y. Liu, X. Yuan, Z. Cui, X.J. Yang, Interfaces, Surface Functionalization of Titanium Alloy with miR-29b Nanocapsules To Enhance Bone Regeneration, ACS Appl Mater Interfaces. 8(9) (2016) 5783.

[41] G. Pan, S. Sun, W. Zhang, R. Zhao, W. Cui, F. He, L. Huang, S.-H. Lee, K.J. Shea, Q.J. Shi, Biomimetic design of mussel-derived bioactive peptides for dual-functionalization of titanium-based biomaterials, J Am Chem Soc. 138(45) (2016) 15078-15086.

[42] T. Xin, Y. Gu, R. Cheng, J. Tang, Z. Sun, W. Cui, L. Chen, Inorganic Strengthened Hydrogel Membrane as Regenerative Periosteum, ACS Appl Mater Interfaces. 9(47) (2017). 41168-41180

[43] R. Kumar Saini, L. Prasad Bagri, A.K. Bajpai, Nano-silver Hydroxyapatite Based Antibacterial 3D Scaffolds of Gelatin/Alginate/Poly (vinyl alcohol) for Bone Tissue Engineering Applications, Colloids Surf B Biointerfaces. 177(1) (2019). 211-218

[44] C. Li, F. Ai, M. Miao, H. Liao, F Li, M. Liu,F. Yu, L. Dong, T Li., X.L. Wang. "The return of ceramic implants": Rose stem inspired dual layered modification of ceramic scaffolds with improved mechanical and anti-infective properties, Mater Sci Eng C Mater Biol Appl. (2018). 873-879.

[45] M.Z. Yao, M.Y. Huang-Fu, H.N. Liu, X.R. Wang, X. Sheng, J.Q. Gao, Fabrication and characterization of drug-loaded nano-hydroxyapatite/polyamide 66 scaffolds modified with carbon nanotubes and silk fibroin, Int J Nanomedicine 11 (2016) 6181-6194.

[46] N. Dehnavi, K. Parivar, V. Goodarzi, A. Salimi, M.R. Nourani, Systematically engineered electrospun conduit based on PGA/collagen/bioglass nanocomposites: The evaluation of morphological, mechanical, and bio-properties, Polym. Adv. Tech. 30(9) (2019) 2192-2206.

[47] A. Abbushi, M. Endres, M. Cabraja, S.N. Kroppenstedt, U.W. Thomale, M. Sittinger, A.A. Hegewald, L. Morawietz, A.J. Lemke, V.G.J.S. Bansemer, Regeneration of intervertebral disc tissue by resorbable cell-free polyglycolic acid-based implants in a rabbit model of disc degeneration, Spine. 33(14) (2008) 1527.

[48] M. Charles-Harris, M.A. Koch, M. Navarro, D. Lacroix, E. Engel, J.A. Planell, A $\mathrm{PLA} /$ calcium phosphate degradable composite material for bone tissue engineering: an in vitro study, J Mater. Sci. 19(4) (2008) 1503-1513. 
[49] M.O. Montjovent, S. Mark, L. Mathieu, C. Scaletta, A. Scherberich, C. Delabarde, P.Y. Zambelli, P.E. Bourban, L.A. Applegate, D.P.J.B. Pioletti, Human fetal bone cells associated with ceramic reinforced PLA scaffolds for tissue engineering, Bone. 42(3) (2008) 554-564.

[50] R. Thakur, C. Florek, J. Kohn, B.J. Michniak, Electrospun nanofibrous polymeric scaffold with targeted drug release profiles for potential application as wound dressing, Int. J Pharm. 364(1) (2008) 87-93.

[51] Y. Murakami, M. Yokoyama, T. Okano, H. Nishida, Y. Tomizawa, M. Endo, H.J. Kurosawa, A novel synthetic tissue-adhesive hydrogel using a crosslinkable polymeric micelle, J Biomed Mater Res A. 80(2) (2007) 421-427.

[52] A. Louvrier, E. Euvrard, L. Nicod, G. Rolin, F. Gindraux, L. Pazart, C. Houdayer, P.Y. Risold, F. Meyer, C. Meyer, Odontoblastic differentiation of dental pulp stem cells from healthy and carious teeth on an original PCL-based 3D scaffold, Int. Endod. J. (2018) e252-e263

[53] A. Shahin-Shamsabadi, A. Hashemi, M. Tahriri, F. Bastami, M. Salehi, F. Mashhadi Abbas, Mechanical, material, and biological study of a PCL/bioactive glass bone scaffold: Importance of viscoelasticity, Mater Sci Eng C Mater Biol Appl. 90 (2018) 280-288.

[54] P. Liu, L. Sun, P. Liu, W. Yu, Q. Zhang, W. Zhang, J. Ma, P. Liu, J. Shen, Surface modification of porous PLGA scaffolds with plasma for preventing dimensional shrinkage and promoting scaffold-cell/tissue interactions, J Mater Chem B 46 (2018). [55] K.S. Park, B.J. Kim, E. Lih, W. Park, S.H. Lee, Y.K. Joung, D.K. Han, Versatile effects of magnesium hydroxide nanoparticles in PLGA scaffold-mediated chondrogenesis, Acta Biomater 73 (2018). 204-216

[56] X. Yang, Z. Lu, H. Wu, W. Li, L. Zheng, J. Zhao, Collagen-alginate as bioink for three-dimensional (3D) cell printing based cartilage tissue engineering, Mater Sci Eng C Mater Biol Appl. 83 (2018) 195-201.

[57] M. Achilli, D.J.P. Mantovani, Tailoring Mechanical Properties of Collagen-Based Scaffolds for Vascular Tissue Engineering: The Effects of $\mathrm{pH}$, Temperature and Ionic Strength on Gelation, Polymers. 2(4) (2010) 664-680.

[58] E.S. Sani, R. Portillo-Lara, A. Spencer, W. Yu, N. Annabi, Engineering Adhesive and Antimicrobial Hyaluronic Acid/Elastin-like Polypeptide Hybrid Hydrogels for Tissue Engineering Applications, Acs Biomat Sci Eng 4(7) (2018). 2528-2540

[59] J. Kim, I.S. Kim, T.H. Cho, H.C. Kim, S.J. Yoon, J. Choi, Y. Park, K. Sun, S.J. Hwang, In vivo evaluation of MMP sensitive high-molecular weight HA-based hydrogels for bone tissue engineering, J Biomed Mater Res A 95A(3) (2010) 673-681.

[60] M.E. Frohbergh, A. Katsman, G.P. Botta, P. Lazarovici, C.L. Schauer, U.G. Wegst, P.I.J.B. Lelkes, Electrospun hydroxyapatite-containing chitosan nanofibers crosslinked with genipin for bone tissue engineering, Biomaterials 33(36) (2012) 9167-9178.

[61] K. Balagangadharan, S.V. Chandran, B. Arumugam, S. Saravanan, N. Selvamurugan, Chitosan/nano-hydroxyapatite/nano-zirconium dioxide scaffolds with miR-590-5p for bone regeneration, Int J Biol Macromol. 111 (2018) 953-958.

[62] P.A. Levett, F.P. Melchels, K. Schrobback, D.W. Hutmacher, J. Malda, T.J. Klein, A 
biomimetic extracellular matrix for cartilage tissue engineering centered on photocurable gelatin, hyaluronic acid and chondroitin sulfate, Acta Biomater. 10(1) (2014) 214-223.

[63] R. Cheng, Y. Yan, H. Liu, H. Chen, G. Pan, L. Deng, W.G. Cui, Mechanically enhanced lipo-hydrogel with controlled release of multi-type drugs for bone regeneration, Appli. Mater. Today 12 (2018) 294-308.

[64] A. Coombes, E. Verderio, B. Shaw, X. Li, M. Griffin, S.J.B. Downes, Biocomposites of non-crosslinked natural and synthetic polymers, Biomaterials. 23(10) (2002) 2113-2118.

[65] F. Du, H. Wang, W. Zhao, D. Li, D. Kong, J. Yang, Y.J.B. Zhang, Gradient nanofibrous chitosan/poly $\varepsilon$-caprolactone scaffolds as extracellular microenvironments for vascular tissue engineering, Biomaterials 33(3) (2012) 762-770.

[66] W. Dai, N. Kawazoe, X. Lin, J. Dong, G.J.B. Chen, The influence of structural design of PLGA/collagen hybrid scaffolds in cartilage tissue engineering, Biomaterials. 31(8) (2010) 2141-2152.

[67] K.O. Kim, Y. Akada, W. Kai, B.S. Kim, I.S.J.J.o.B. Kim, Nanobiotechnology, Cells Attachment Property of PVA Hydrogel Nanofibers Incorporating Hyaluronic Acid for Tissue Engineering, 2(4) (2011) 353-360.

[68] Z. Meng, W. Zheng, L. Li, Y.J.M.C. Zheng, Physics, Fabrication, characterization and in vitro drug release behavior of electrospun PLGA/chitosan nanofibrous scaffold, Mater Chem Phys 125(3) (2011) 606-611.

[69] W. Zhu, Y. Li, L. Liu, Y. Chen, F.J. Xi, Supramolecular hydrogels as a universal scaffold for stepwise delivering Dox and Dox/cisplatin loaded block copolymer micelles, Int J Pharm 437(1-2) (2012) 11-19.

[70] X. Liu, X. Zhang, M. Zhu, G. Lin, J. Liu, Z. Zhou, X. Tian, Y. Pan, PEGylated Au@ Pt nanodendrites as novel theranostic agents for computed tomography imaging and photothermal/radiation synergistic therapy, ACS Appl Mater Interfaces. 9(1) (2016) 279-285.

[71] W. Hu, X. Wei, L. Zhu, D. Yin, A. Wei, X. Bi, T. Liu, G. Zhou, Y. Qiang, X.J.N.E. Sun, Enhancing proliferation and migration of fibroblast cells by electric stimulation based on triboelectric nanogenerator, Nano Energy. 57 (2019) 600-607.

[72] S.G. Antimisiaris, D. Siablis, E. Liatsikos, C. Kalogeropoulou, I. Tsota, V. Tsotas, D. Karnabatidis, D.G. Fatouros, G.A. Barbalias, Liposome-coated metal stents: an in vitro evaluation of controlled-release modality in the ureter, J Endourol. 14(9) (2000) 743-7.

[73] F. Wang, J. Liu, Liposome supported metal oxide nanoparticles: interaction mechanism, light controlled content release and intracellular delivery, Small. 15;10(19) (2014). 3927-31

[74] F. Wang, J. Liu, A stable lipid/TiO2 interface with headgroup-inversed phosphocholine and a comparison with SiO2, J Am Chem Soc. 137(36) (2015) 11736-11742.

[75] C. Wu, Y. Cong, M. Chu, A gold nanoshell with a silica inner shell synthesized using liposome templates for doxorubicin loading and near-infrared photothermal 
therapy, Int J Nanomedicine (2011) 807-813.

[76] Y. He, M. Li, W. Jiang, W.J. Yang, L. Lin, L.J. Xu, F.J.J.o.M.C.B. Fu, Phosphatidylserine-functionalized Fe 304@SiO 2 nanoparticles combined with enzyme encapsulated liposome for the visual detection of $\mathrm{Cu} 2+, \mathrm{J}$ Mater. Chem. B 4(4) (2016) 752-759.

[77] J.J.B. Kopeček, Hydrogel biomaterials: a smart future?, Biomaterials. 28(34) (2007) 5185-5192.

[78] A. Suri, C. Rui, D.G. Rackus, N.J.S. Spiller, C. Richardson, L.O. Pålsson, R.J.S.M. Kataky, Liposome-doped hydrogel for implantable tissue, Soft. Matter. 7(15) (2011) 7071-7077.

[79] S. Kazakov, M. Kaholek, D. Kudasheva, I. Teraoka, M.K.C. And, K.J.L. Levon, Poly(N-isopropylacrylamide-co-1-vinylimidazole) Hydrogel Nanoparticles Prepared and Hydrophobically Modified in Liposome Reactors: Atomic Force Microscopy and Dynamic Light Scattering Study, Langmuir 19(19) (2015) 8086-8093.

[80] S. Mourtas, M. Haikou, M. Theodoropoulou, C. Tsakiroglou, S.G. Antimisiaris, The effect of added liposomes on the rheological properties of a hydrogel: $A$ systematic study, J Colloid Interface Sci. 317(2) (2008) 611-619.

[81] W. Gao, D. Vecchio, J. Li, J. Zhu, Q. Zhang, V. Fu, J. Li, S. Thamphiwatana, D. Lu, L. Zhang, Hydrogel Containing Nanoparticle-Stabilized Liposomes for Topical Antimicrobial Delivery, ACS Nano 8(3) (2014) 2900-7.

[82] A.S. Hoffman, Hydrogels for biomedical applications, Ann N Y Acad Sci. 64 (2012) 18-23.

[83] E.L.G. Ruel-Gariepy, P. Hildgen, A. Gupta, J.C. Leroux, Thermosensitive chitosan-based hydrogel containing liposomes for the delivery of hydrophilic molecules, J Control Release. 82(2-3) (2002) 373-383.

[84] Y.H. Ma, J. Yang, B. Li, Y.W. Jiang, X. Lu, Z.J. Chen, Biodegradable and injectable polymer-liposome hydrogel: A promising cell carrier, Poly. Chem. 7(11) (2016) 2037-2044.

[85] R. Li, Q. Liu, H. Wu, K. Wang, L. Li, C. Zhou, N.J. Ao, Preparation and characterization of in-situ formable liposome/chitosan composite hydrogels, Materials Letters 220 (2018) 289-292.

[86] H.S. O'Neill, C.C. Herron, C.L. Hastings, R. Deckers, A.L. Noriega, H.M. Kelly, W.E. Hennink, C.O. McDonnell, F.J. O’Brien, E.J. Ruiz-Hernández, GP Duffy. A stimuli responsive liposome loaded hydrogel provides flexible on-demand release of therapeutic agents, Acta Biomater. 48 (2017) 110-119.

[87] J. Doshi, D.H. Reneker, Electrospinning process and applications of electrospun fibers, J electrostatics 35(2-3) (1995) 151-160.

[88] S.A. Sell, P.S. Wolfe, K. Garg, J.M. McCool, I.A. Rodriguez, G.L.J.P. Bowlin, The use of natural polymers in tissue engineering: a focus on electrospun extracellular matrix analogues, Polymers 2(4) (2010) 522-553.

[89] J.M. Deitzel, J. Kleinmeyer, D. Harris, N.B.J.P. Tan, The effect of processing variables on the morphology of electrospun nanofibers and textiles, Polymer 42(1) (2001) 261-272.

[90] Li. Z, K. H, C. N, L. Z, L. P, L. W, Z. C, C. C, L. R, H.Y.J.C. Polymers, Controlled 
Release of Liposome-Encapsulated Naproxen from Core-sheath Electrospun Nanofibers, Carbohyd Polym. 111(1) (2014) 18-24.

[91] L. Lin, Y. Dai, H. Cui, Antibacterial poly (ethylene oxide) electrospun nanofibers containing cinnamon essential oil/beta-cyclodextrin proteoliposomes, Carbohydr Polym. 178 (2017) 131-140.

[92] R. Chandrawati, M.T. Olesen, T.C. Marini, G. Bisra, A.G. Guex, M.G. de Oliveira, A.N. Zelikin, M.M. Stevens, Enzyme prodrug therapy engineered into electrospun fibers with embedded liposomes for controlled, localized synthesis of therapeutics, Adv Healthc Mater. 6(17) (2017) 1700385.

[93] N. Monteiro, A. Martins, L.R. Rui, N.M. Neves, Liposomes in tissue engineering and regenerative medicine, J R Soc Interface. 11(101) (2014) 20140459.

[94] M. Batory, A. Adamus-Grabicka, A. Sobczyk-Guzenda, N. Bartoszek, P. Komorowski, L. Vyslouzilova, Z. Rozek, E.J. Budzisz, The use of liposomes in the modification of polycaprolactone fibers, Appli. Polymer. 133(15) (2016).

[95] D.G. Yu, C. Branfordwhite, G.R. Williams, S.W.A. Bligh, K. White, L.M. Zhu, N. Chatterton, Self-assembled liposomes from amphiphilic electrospun nanofibers, Pharmaceutics. 7(18) (2011) 8239-8247.

[96] M. Gupta, A.K. Goyal, S.R. Paliwal, R. Paliwal, N. Mishra, B. Vaidya, D. Dube, S.K. Jain, S.P. Vyas, Development and characterization of effective topical liposomal system for localized treatment of cutaneous candidiasis, J Liposome Res. 20(4) (2010) 341-350.

[97] J.L. Larson, T.L. Wallace, R.W. Tyl, M.C. Marr, C.B. Myers, P.A. Cossum, The reproductive and developmental toxicity of the antifungal drug Nyotran ${ }^{\circledR}$ (liposomal nystatin) in rats and rabbits, Toxic. Sci. 53(2) (2000) 421-429.

[98] F. Ambrosch, G. Wiedermann, S. Jonas, B. Althaus, B. Finkel, R. Glück, C.J.V. Herzog, Immunogenicity and protectivity of a new liposomal hepatitis A vaccine, Vaccine. 15(11) (1997) 1209-1213.

[99] A. Hamada, T. Kawaguchi, M.J.C.p. Nakano, Clinical pharmacokinetics of cytarabine formulations, Clin Pharmacokinet. 41(10) (2002) 705-718.

[100] Y. Mao, L. Xue, C. Ge, S.J. Wang, Thermosensitive Hydrogel System with Paclitaxel Liposomes Used in Localized Drug Delivery System for In Situ Treatment of Tumor: Better Antitumor Efficacy and Lower Toxicity, J Pharm Sci. 105(1) (2015) 194-204.

[101] Y. Xing, H. Chen, S. Li, X.J. Guo, In vitro and in vivo investigation of a novel two-phase delivery system of 2-methoxyestradiol liposomes hydrogel, J Liposome Res. 24(1) (2014) 10-16.

[102] R. Bai, X. Deng, Q. Wu, X. Cao, T. Ye, S.J. Wang, Technology, Liposome-loaded thermo-sensitive hydrogel for stabilization of SN-38 via intratumoral injection: optimization, characterization and antitumor activity, J. Pharm. Deve. Tech. 23(1)(2018)

[103] N. Zhang, H. Chen, A.Y. Liu, J.J. Shen, V. Shah, C. Zhang, J. Hong, Y. Ding, Gold conjugate-based liposomes with hybrid cluster bomb structure for liver cancer therapy, Biomaterials. 74 (2016) 280-291.

[104] A.L. Byrd, Y. Belkaid, J.A. Segre, The human skin microbiome, Nat Rev 
Microbiol. 16(3) (2018) 1177-1179.

[105] X. Gong, Y. Yang, L. Huang, Q. Zhang, R.-Z. Wan, P. Zhang, B. Zhang, Antioxidation, anti-inflammation and anti-apoptosis by paeonol in LPS/d-GalN-induced acute liver failure in mice, Int Immunopharmacol. 46 (2017) 124-132.

[106] H. Xia, Z. Cheng, Y.J.L.A.J.o.P. Xu, Paeonol Liposome-Hydrogel: Preparation, Penetration Through the Mouse Skin and Down-Regulation of the Expression of Tyrosinase, Latin American J Pharm. 33(8) (2014) 1267-1272.

[107] A.K. Jain, A. Jain, N.K. Garg, A. Agarwal, A. Jain, S.A. Jain, R.K. Tyagi, R.K. Jain, H. Agrawal, G.P.J.C. Agrawal, S.B. Biointerfaces, Adapalene loaded solid lipid nanoparticles gel: an effective approach for acne treatment, Colloids Surf B Biointerfaces. 121 (2014) 222-229.

[108] S.K. Mandlik, S.S. Siras, K.R. Birajdar, Optimization and characterization of sertaconazole nitrate flexisomes embedded in hydrogel for improved antifungal activity, J Liposome Res. 29 (1) (2019) 10-20.

[109] M.R. Haque, D.Y. Lee, C.H. Ahn, J.H. Jeong, Y. Byun, Local co-delivery of pancreatic islets and liposomal clodronate using injectable hydrogel to prevent acute immune reactions in a type 1 diabetes, Pharm Res. 31(9) (2014) 2453-62.

[110] X. Chen, B.C.K. Wong, H. Chen, S. Zhang, Z. Bian, G. Zhang, C. Lin, M.K. Riaz, D. Tyagi, A. Lu, Long-lasting Insulin Treatment Via a Single Subcutaneous Administration of Liposomes in Thermoreversible Pluronic ${ }^{\circledR}$ F127 Based Hydrogel, Curr Pharm Des. 23(39) (2018) 6079-6085.

[111] P.A. Efron, F.A. Moore, S.C. Brakenridge, Persistent Inflammation, Immunosuppression and Catabolism after Severe Injury or Infection, Annual Update in Intensive Care and Emergency Medicine 2018. pp 25-35

[112] R. Medzhitov, Origin and physiological roles of inflammation, Nature. 454(7203) (2008) 428.

[113] P. Negi, M. Aggarwal, G. Sharma, C. Rathore, G. Sharma, B. Singh, O.P.J.B. Katare, Niosome-based hydrogel of resveratrol for topical applications: An effective therapy for pain related disorder(s), Biomedicine. Pharm. 88 (2017) 480-487.

[114] L. Ryom, C. Boesecke, M. Bracchi, J. Ambrosioni, E. Martínez, Highlights of the 2017 European AIDS Clinical Society (EACS) Guidelines for the treatment of adult HIV-positive persons version 9.0, HIV Med. 19(5) (2018). 309-315

[115] R. Ramanathan, Y. Jiang, B. Read, S. Golan-Paz, K.A Woodrow, Biophysical characterization of small molecule antiviral-loaded nanolipogels for HIV-1 chemoprophylaxis and topical mucosal application, Acta Biomater. 36 (2016) 122-131.

[116] J. Zhu, W. Xiong, X.U. Infection, Advances in research of the pathogenesis of norovirus, J Clin. Pediatrics. 36(3) (2018). 231-234

[117] J. Hurler, K.K. Sørensen, A. Fallarero, P. Vuorela, N.J.B.r.i. Škalko-Basnet, Liposomes-in-hydrogel delivery system with mupirocin: in vitro antibiofilm studies and in vivo evaluation in mice burn model, Biomed Res Int. 2013 (2013). :498485

[118] P. Leucht, S. Lee, N. Yim, Wnt signaling and bone regeneration: Can't have one without the other, Biomaterials 196 (2018). 46-50 
[119] J.D. Boerckel, Y.M. Kolambkar, H.Y. Stevens, A.S. Lin, K.M. Dupont, R.E. Guldberg, Effects of in vivo mechanical loading on large bone defect regeneration, J Orthop Res. 30(7) (2012) 1067-1075.

[120] M. Mohammadi, M. Alibolandi, K. Abnous, Z. Salmasi, M.R. Jaafari, M. Ramezani, Biology, Medicine, Fabrication of hybrid scaffold based on hydroxyapatite-biodegradable nanofibers incorporated with liposomal formulation of BMP-2 peptide for bone tissue engineering, Nanomedicine 14(7) (2018) 1987-1997.

[121] Z.K. Cui, S. Kim, J.J. Baljon, M. Doroudgar, M. Lafleur, B.M. Wu, T. Aghaloo, L. Min, Design and Characterization of a Therapeutic Non-phospholipid Liposomal Nanocarrier with Osteoinductive Characteristics To Promote Bone Formation, ACS Nano. 11(8) (2017) 8055-8063.

[122] H. Chen, Y. Yan, J. Qi, L. Deng, W. Cui, Sustained delivery of desferrioxamine via liposome carriers in hydrogel for combining angiogenesis and osteogenesis in bone defects reconstruction, J Control Release 259 (2017) e79.

[123] F. Zarei, M. Soleimaninejad, Role of growth factors and biomaterials in wound healing, Artif Cells Nanomed Biotechnol. 46(sup1) (2018) 906-911

[124] H.H. Homann, O. Rosbach, W. Moll, P.M. Vogt, G. Germann, M. Hopp, B. Langer-Brauburger, K. Reimer, H.U. Steinau, A liposome hydrogel with polyvinyl-pyrrolidone iodine in the local treatment of partial-thickness burn wounds, Ann Plast Surg. 59(4) (2007) 423-427.

[125] H.L. Xu, P.P. Chen, D.L. Zhuge, Q.Y. Zhu, B.H. Jin, B.X. Shen, J. Xiao, Y.Z. Zhao, Liposomes with Silk Fibroin Hydrogel Core to Stabilize bFGF and Promote the Wound Healing of Mice with Deep Second-Degree Scald, Adv Healthc Mater 6(19) (2017).

[126] J. Hurler, O.A. Berg, M. Skar, A.H. Conradi, P.J. Johnsen, N. Skalkobasnet, Improved burns therapy: liposomes-in-hydrogel delivery system for mupirocin, J Pharm Sci. 101(10) (2012) 3906-3915.

[127] G. Ziegler, P. Grabher, A. Thompson, D. Altmann, M. Hupp, J. Ashburner, K. Friston, N. Weiskopf, A. Curt, P. Freund, Progressive neurodegeneration following spinal cord injury: Implications for clinical trials, Neurology 90(14) (2018). :e1257-e1266.

[128] X. Li, J. Dai, Bridging the gap with functional collagen scaffolds: tuning endogenous neural stem cells for severe spinal cord injury repair, Biomater Sci. 6 (2018). 265-271

[129] X. Li, C. Fan, Z. Xiao, Y. Zhao, H. Zhang, J. Sun, Y. Zhuang, X. Wu, J. Shi, Y. Chen, A collagen microchannel scaffold carrying paclitaxel-liposomes induces neuronal differentiation of neural stem cells through Wnt/ $\beta$-catenin signaling for spinal cord injury repair, Biomaterials 183 (2018) 114-127.

[130] G.E. Melling, J.S. Colombo, S.J. Avery, W.N. Ayre, S.L. Evans, R.J. Waddington, A.J. Sloan, Liposomal delivery of demineralized dentin matrix for dental tissue regeneration, Tissue Eng Part A. 24(13-14) (2018) 1057-1065. 
(A)

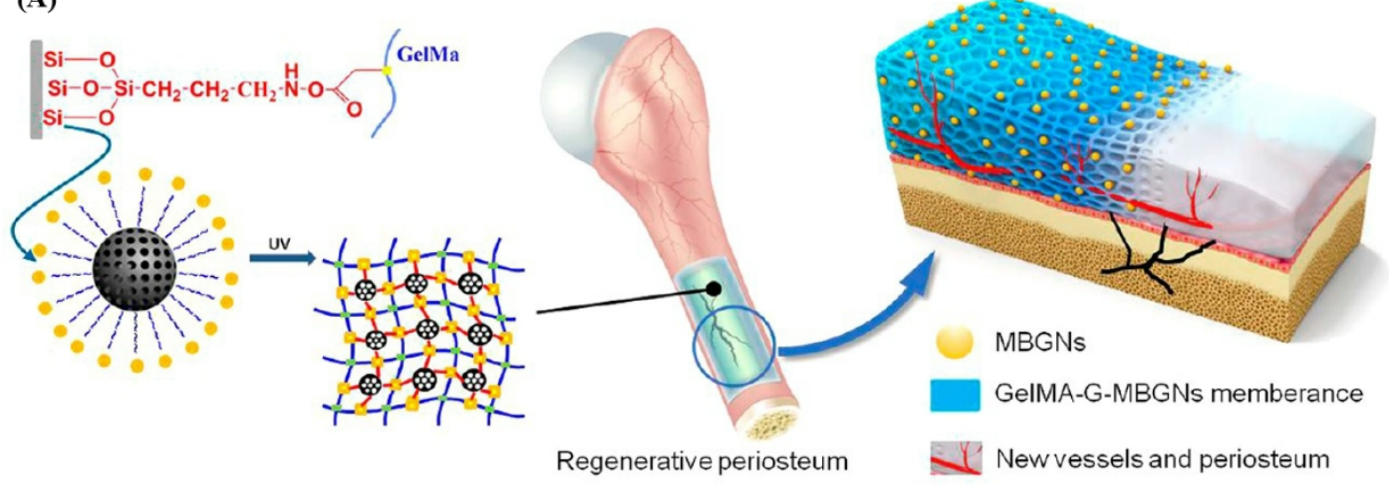

(B)

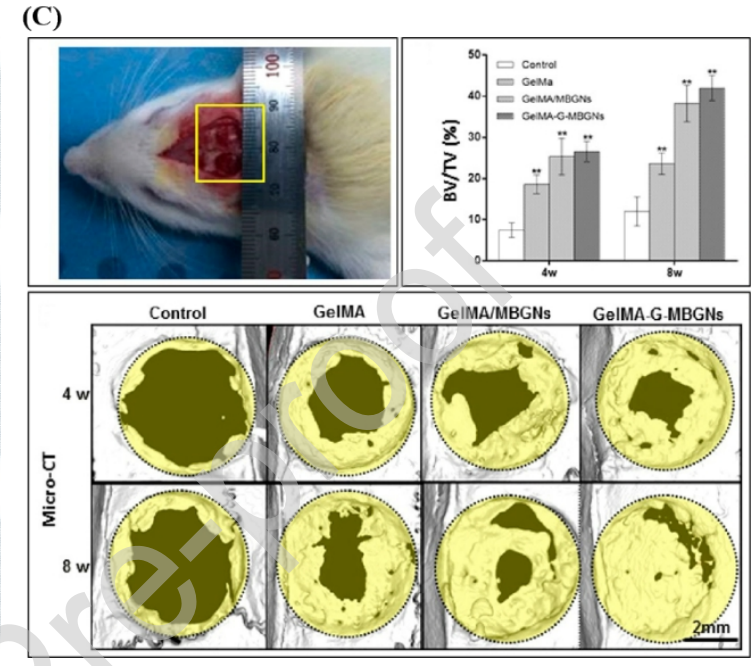

Figure 1. (A) Scheme of inorganic strengthened hydrogel membrane for regenerative periosteum. (B) Progress of fabricating methacrylic acid modified gelatin, amino modified MBGNs and GelMA-MBGNs(G-MBGNs) and preparing GelMA/MBGNs and GeIMA-G-MBGNs. (C) Regenerative periosteum utilized in treating calvarial critical-sized defects in Sprague Dawley rats. Reprinted with permission from ref. [49]. 


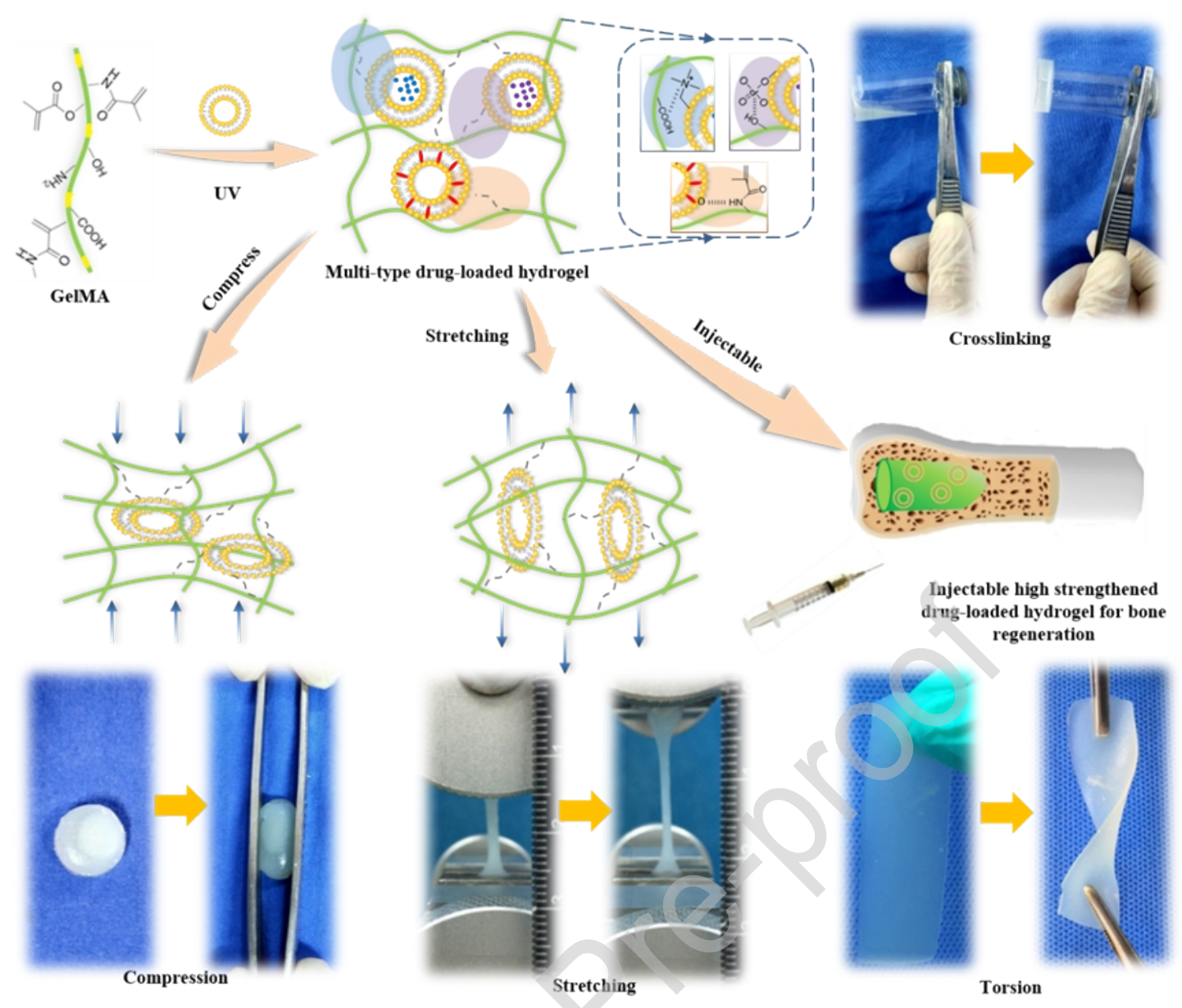

Figure 2. Scheme of mechanically enhanced lipo-hydrogel with controlled release of multi-type drugs for bone regeneration. 


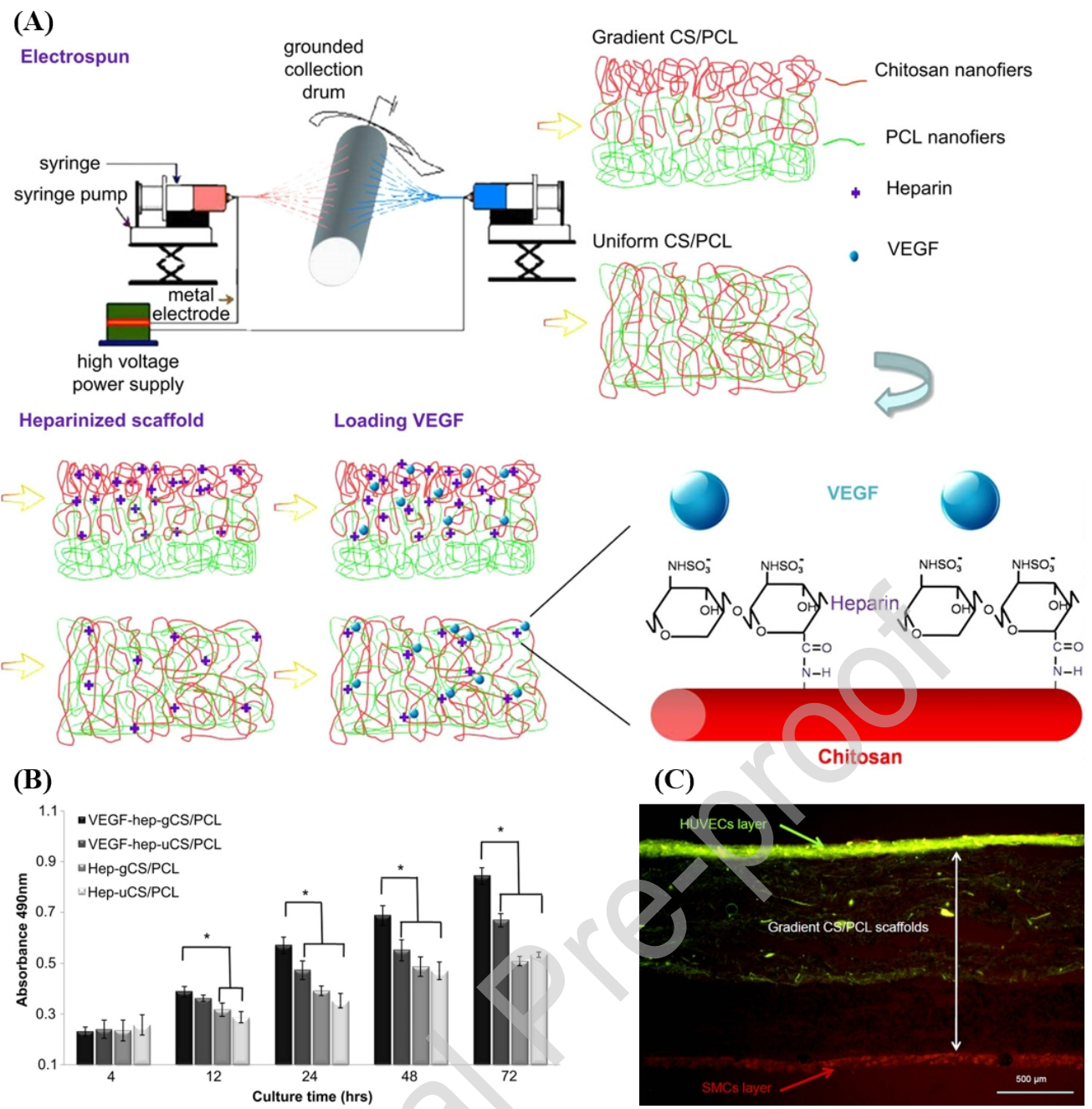

Figure 3. (A) Schematic diagram of gradient and uniform nano-fibrous scaffolds for vascular tissue regeneration. (B) The proliferation of human umbilical vein endothelial cells (HUVECS) on the nanofibrous scaffolds with different formulation $i$ by MTT assay. (C) Fluorescent images of cross-section of sandwich cell-scaffold-cell structure. Reprinted with permission from ref. [65]. 
(A)
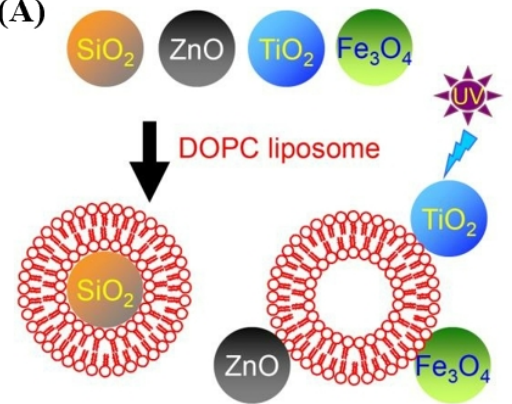

(D)

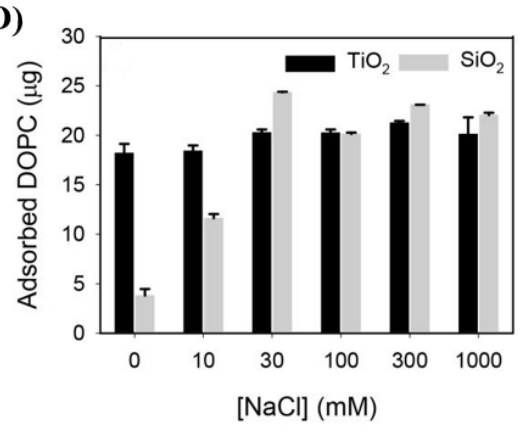

(G)

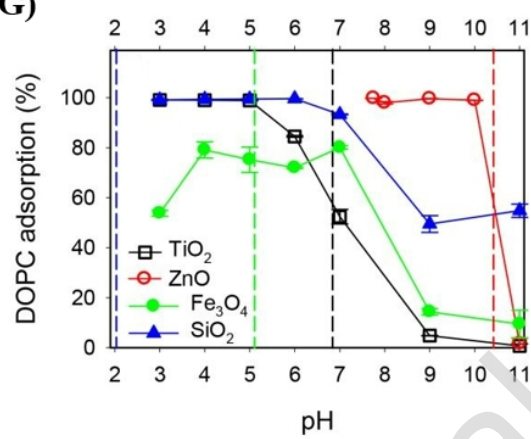

(B)

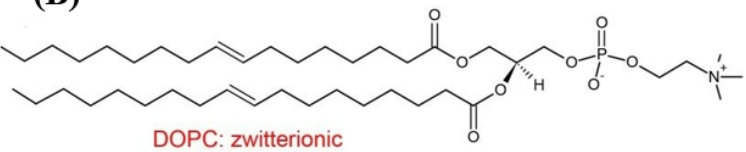

(C) Rh-DOPC $\quad \mathrm{TiO}_{2} \quad \mathrm{ZnO} \quad \mathrm{SiO}_{2} \quad \mathrm{Fe}_{3} \mathrm{O}_{4}$

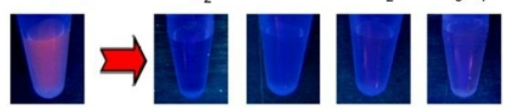

(E)

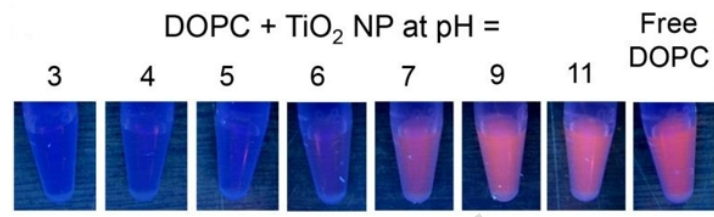

(F)

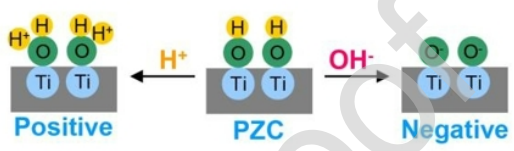

(H)

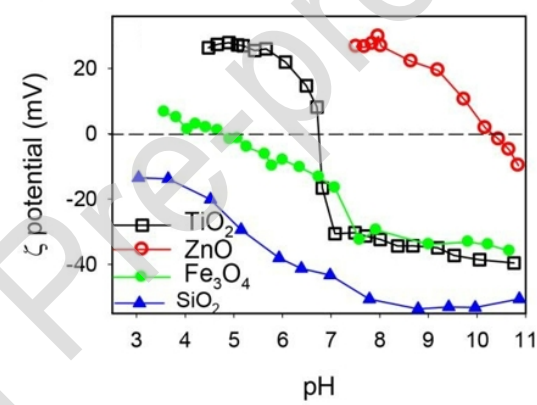

Figure 4. (A) Hybrid nanostructure formed using the oxides and DOPC liposomes. $\mathrm{SiO}_{2}$ NPs form supported bilayers, while the other oxides are adsorbed. $\mathrm{TiO}_{2}$ is a photocatalyst. (B) Chemical structure of the DOPC lipid. (C) Rh-DOPC liposome adsorption by the oxides indicated by the different supernatant fluorescence intensity. (D) The mass of DOPC associated with $100 \mu \mathrm{g}$ of $\mathrm{TiO}_{2}$ or $\mathrm{SiO}_{2} \mathrm{NPs}$ as a function of $\mathrm{NaCl}$ concentration. (E) Photographs of Rh-labeled DOPC liposome interacting with $\mathrm{TiO}_{2}$ NPs as a function of $\mathrm{pH}$. (F) Scheme of protonation of $\mathrm{TiO}_{2}$, affecting its surface charge. (G) Quantification of DOPC adsorption by various oxides as a function of $\mathrm{Ph} .(\mathrm{H}) \zeta$-potential as a function of $\mathrm{pH}$ for the oxides. Reprinted with permission from ref. [85]. 

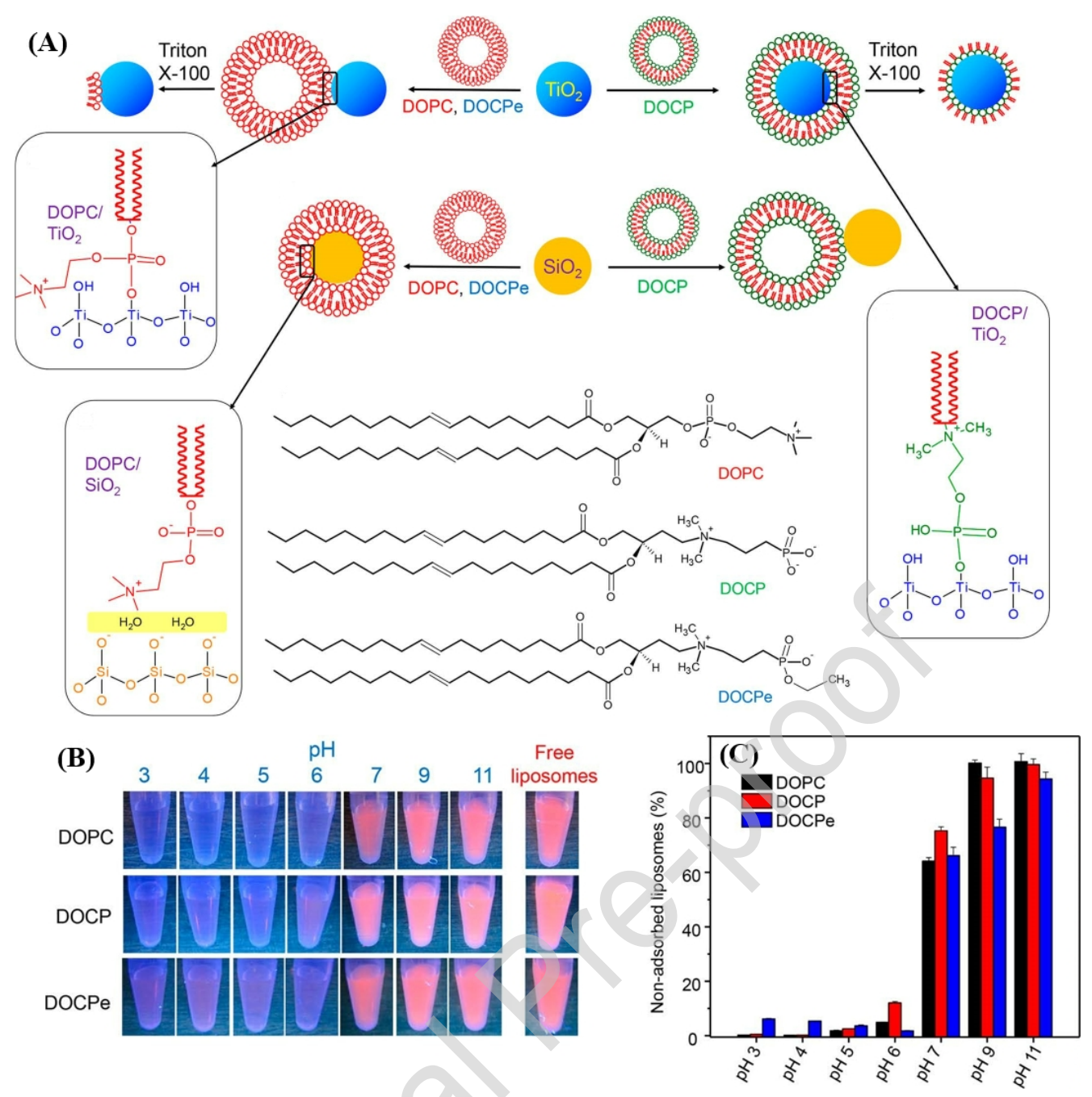

Figure 5. (A) Schematics of DOPC liposome adsorption by $\mathrm{TiO}_{2} \mathrm{NPs}$ and DOCP liposome forming supported bilayers. (B) Photographs of the supernatants of the Rh-labeled DOPC, DOCP, and DOCPe liposomes mixing with $\mathrm{TiO}_{2} \mathrm{NPs}$ at various $\mathrm{pH}$ values and after centrifugation. (C) Quantitative analysis non-adsorbed liposomes by $\mathrm{TiO}_{2}$ as a function of $\mathrm{pH}$. Reprinted with permission from ref. [86]. 


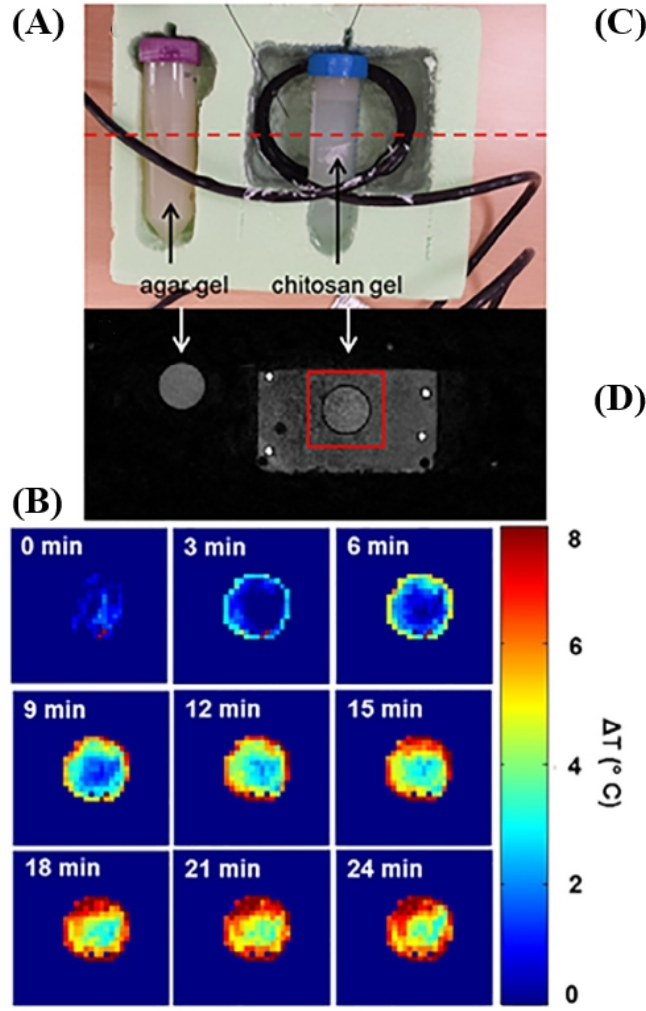

(C)

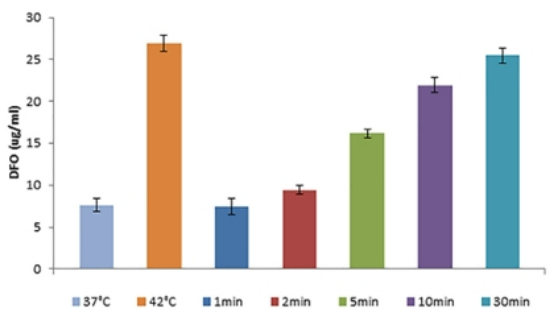

(D)

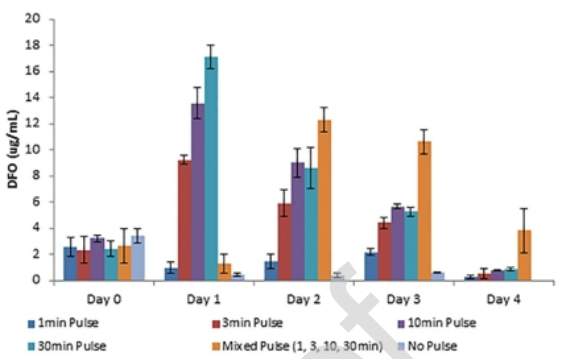

(E)

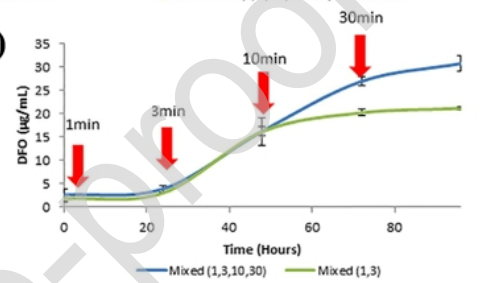

Figure 6. (A) Picture of heating set-up showing the chitosan hydrogel in the water bath and agar gel thermally insulated. (B) coronal temperature maps showing the temperature changes in the chitosan gel at 3 min increments. (C) Absolute DFO release from Lipogel in response to increased durations of hyperthermia. (D) DFO release $(100 \mu \mathrm{M})$ from chitosan/b-GP gels containing liposomal DFO at $37{ }^{\circ} \mathrm{C}$ after a repeated hyperthermic pulse of 1, 3, 10 or $30 \mathrm{~min}$. (E) Mixed pulse Lipogel permitted multiple DFO doses by increasing the duration of hyperthermia every $24 \mathrm{~h}$ (red arrow) $(1,3,10$, and $30 \mathrm{~min})$. Reprinted with permission from ref. [98]. 

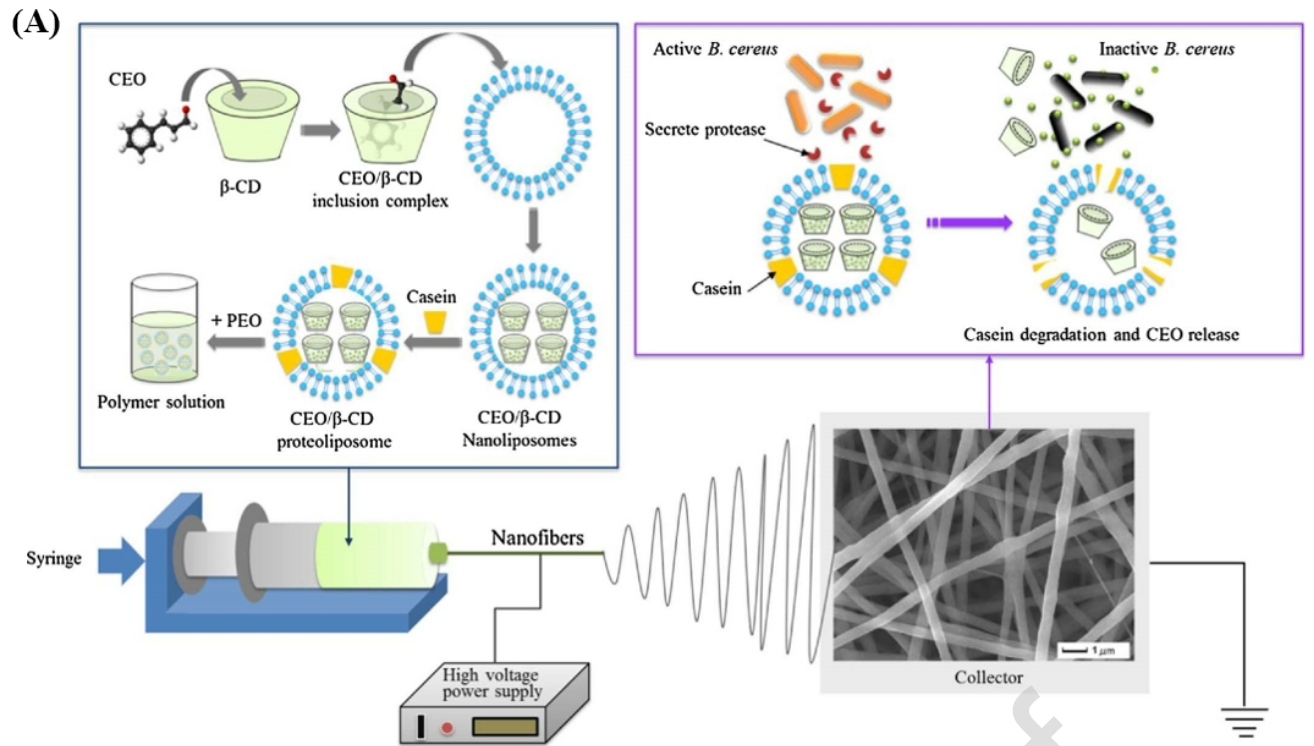

(B)

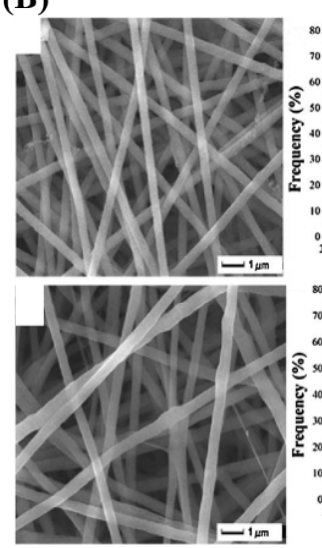

(C)

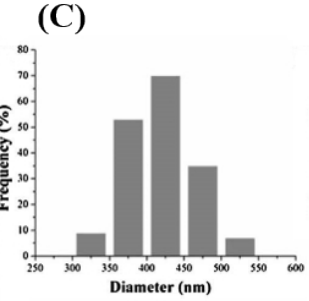

(D)

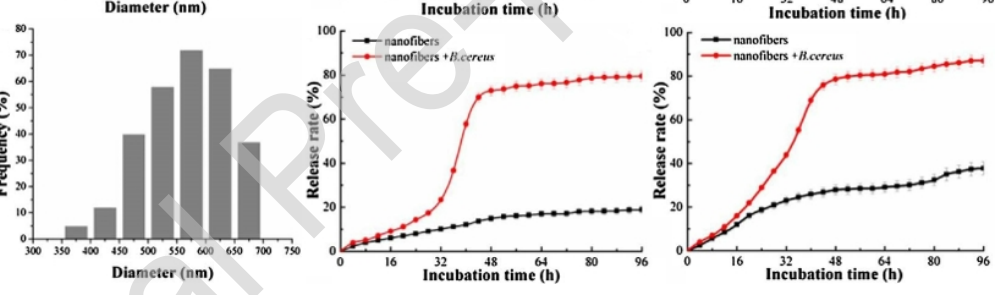

Figure 7. (A) Schematic of electrospinning for $C E O / \beta-C D$ proteoliposomes incorporated into PEO nanofibers. And schematic of $B$. cereus proteinase-triggered CEO release from CEO/ $\beta-C D$ proteoliposomes. (B) The SEM-micrographs of pure PEO nanofibers and CEO/ $\beta-C D$ proteoliposomes nanofibers. (C) Diameter distribution of pure PEO nanofibers and CEO/ $\beta-C D$ proteoliposomes nanofibers. (D) The release rate of $C E O / \beta-C D$ proteoliposomes nanofibers stored at different temperature. Reprinted with permission from ref. [103]. 

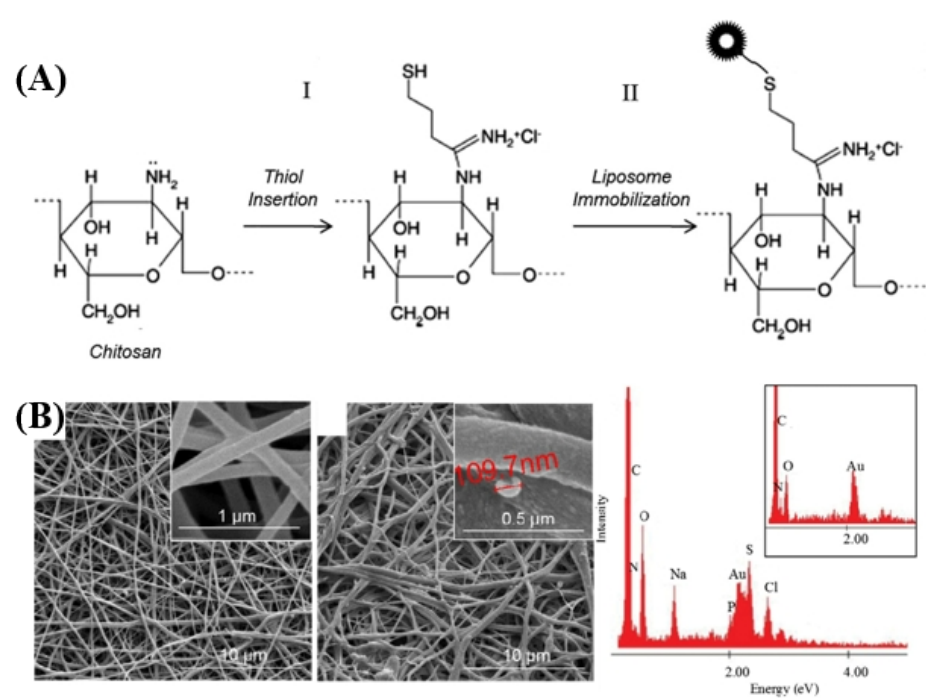

(C)

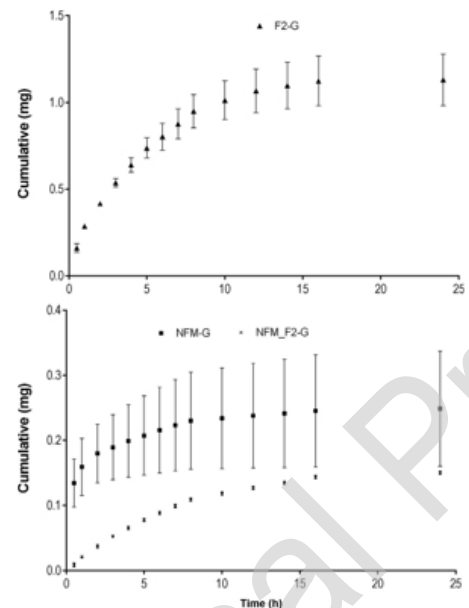

(D)

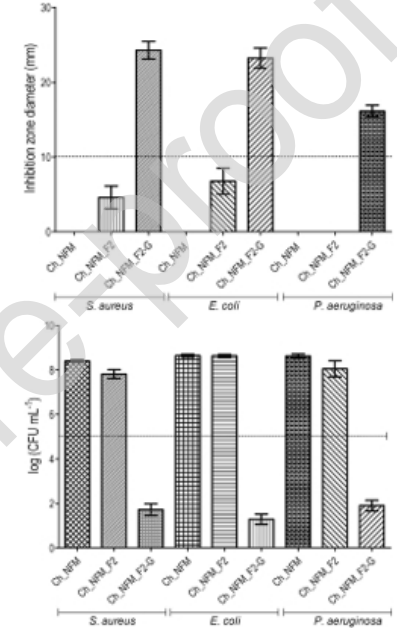

Figure 8. (A) Chemical pathway for the immobilization of liposomes on the surface of Ch NFM. (B) SEM results and EDS spectrum of immobilized Gent-loaded liposomes at the surface of electrospun Ch NFMs. (C) In vitro cumulative release of gentamicin from Ch nanofibers immersed in gentamicin solution, gentamicin release from liposomes in suspension and immobilized at the surface of one electrospun Ch NFM. (D) In vitro antibacterial activity of the corresponding blank control, gentamicin control, electrospun Ch NFM, Gent free liposomes immobilized electrospun Ch NFM, and Gent-loaded liposomes immobilized electrospun Ch NFM against S. aureus, E. coli, and $P$. aeruginosa assessed by the different assay methods. Reprinted with permission from ref. [105]. 


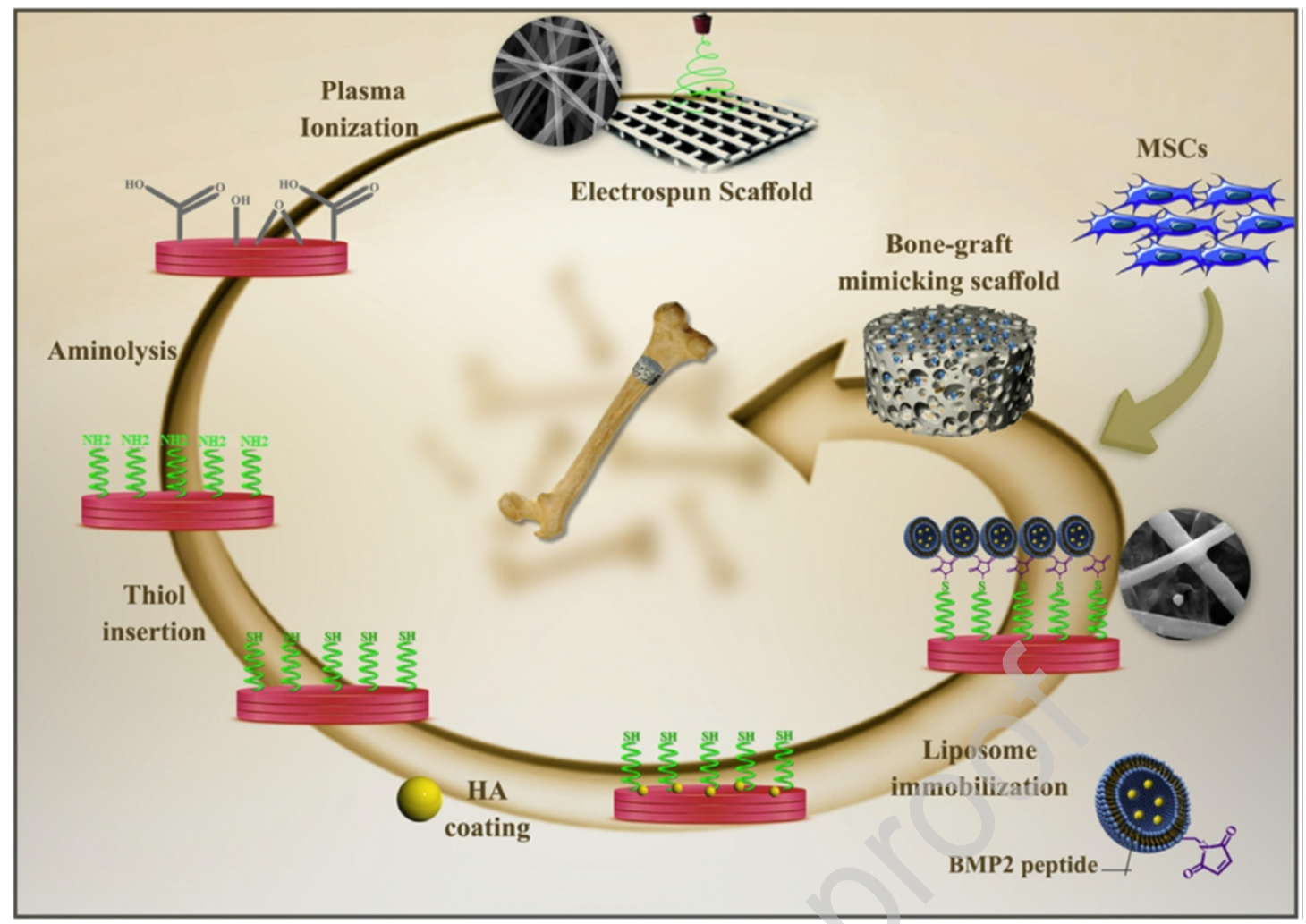

Figure 9. A brief summary illustrating functionalization of the scaffold platform and immobilization of BMP-2 peptide loaded liposomes on to the scaffold to fabricate bone-graft mimicking scaffold for bone tissue engineering. Reprinted with permission from ref. [134]. 


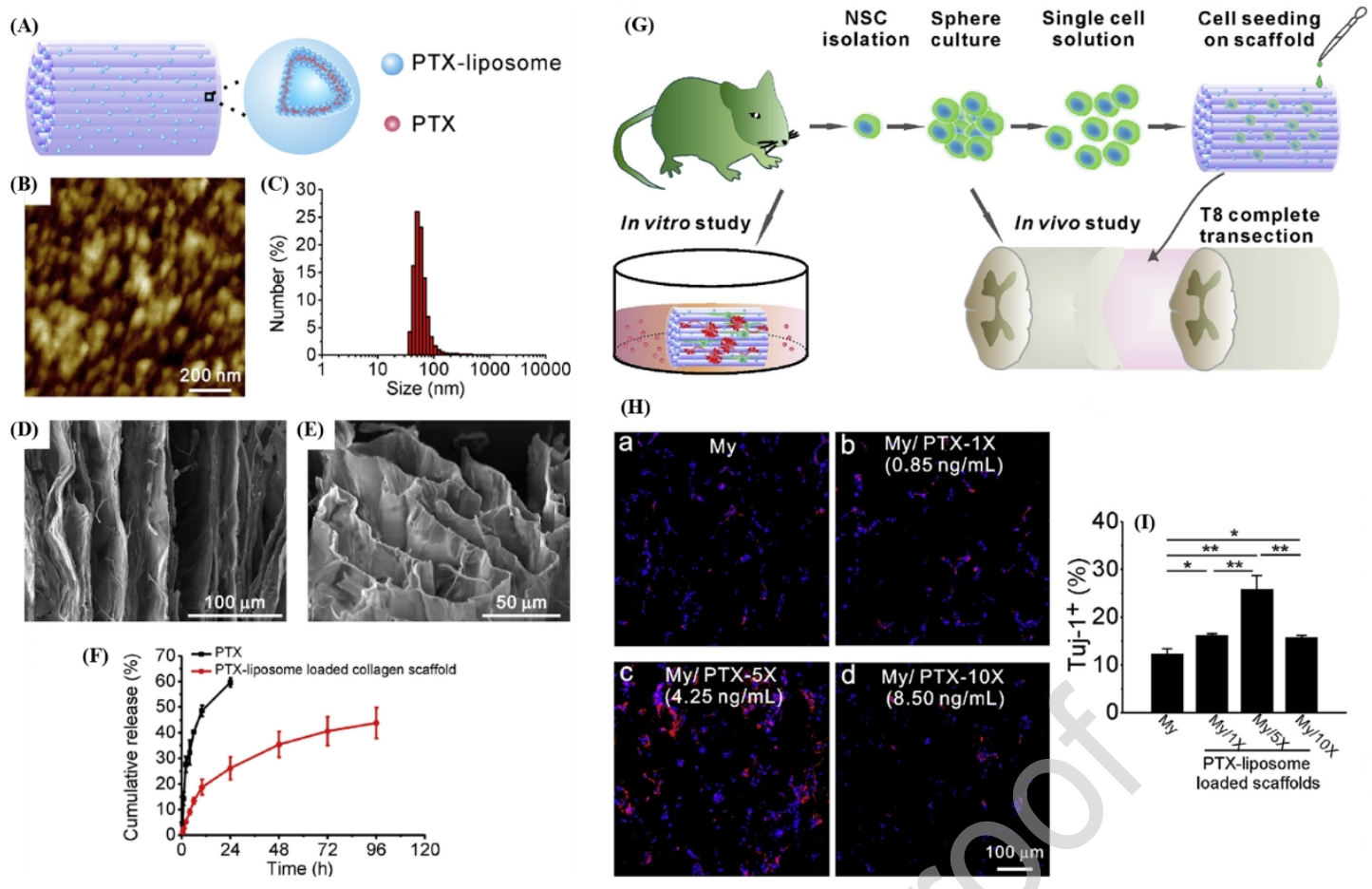

Figure 10. (A) Schematic illustration of a collagen scaffold carrying PTX-liposomes. (B) AFM image of PTX-liposomes. (C) Particle size and particle size distribution of PTX-liposomes. (D, E) SEM images of collagen scaffolds showing the (D) longitudinal and (E) transverse morphologies. (F) Cumulative release profiles of PTX-liposome loaded collagen scaffolds in PBS containing $1.0 \mathrm{M}$ sodium salicylate at $37{ }^{\circ} \mathrm{C}$ compared with pristine PTX. (G) Design of in vitro study and in vivo transplantation at T8 complete transection of rat spinal cord. (H) Representative images of Tuj-1 immunostaining of NSCs cultured on (a) collagen scaffold, and collagen scaffolds carrying varied dose of PTX-liposomes (b) 0.85 (1x), (c) 4.25 (5x), and (d) $8.50 \mathrm{ng} / \mathrm{mL}$ (10x). (I) Quantification of neuronal differentiation rates. Reprinted with permission from ref. [143]. 
Table 1. The fabrication methods of different platforms modified with liposomes.

\begin{tabular}{lcl}
\hline Platform (modified with liposomes) & Fabrication methods & Ref. \\
\hline \multirow{2}{*}{ Mental scaffold } & Surface modification & {$[74][75]$} \\
& Coating & {$[75]$} \\
Inorganic scaffold & Surface modification & {$[76][78]$} \\
& Coating & {$[76][77]$} \\
Hydrogel & Coating & {$[91][96]$} \\
& Mixing & {$[80][82][83][85][88]$} \\
Electrospinning fibers & Surface modification & {$[93][94]$} \\
& Internal loading & {$[90][91][92]$} \\
& Self-assembly & {$[95]$} \\
\hline
\end{tabular}

Table 2. The functions of diverse platforms modified with liposomes in treating diseases.

\begin{tabular}{lcc}
\hline Disease treatment & Platform (modified with liposomes) & Ref. \\
\hline Cancer Therapy & Hydrogel & {$[100][101][102]$} \\
Skin disease & Mental scaffold & {$[103]$} \\
Diabetes & Hydrogel & {$[106][107][108]$} \\
Inflammation & Hydrogel & {$[109][110]$} \\
HIV & Hydrogel & {$[113]$} \\
Infection & Hydrogel & {$[115]$} \\
\hline
\end{tabular}

Table 3. The functions of different types of platforms modified with liposomes in tissue regeneration.

\begin{tabular}{lcc}
\hline \multicolumn{1}{l}{$\begin{array}{l}\text { Tissue } \\
\text { regeneration }\end{array}$} & Platform (modified with liposomes) & Ref. \\
\hline Bone & Electrospinning fibers & {$[120]$} \\
& Hydrogel & {$[121][122]$} \\
Wound healing & Hydrogel & {$[124][125][126]$} \\
Spinal cord & Collagen fibers & {$[129]$} \\
Teeth & Demineralized dentin matrix & {$[130]$} \\
\hline
\end{tabular}




\section{Declaration of interests}

$\bigotimes$ The authors declare that they have no known competing financial interests or personal relationships that could have appeared to influence the work reported in this paper.

$\square$ The authors declare the following financial interests/personal relationships which may be considered as potential competing interests:

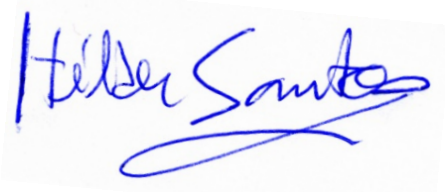

Hélder Santos

(corresponding author, on behalf of all co-authors) 